\title{
Spin Physics through QCD Instantons
}

\author{
Yachao Qian ${ }^{1}$ and Ismail Zahed ${ }^{1}$ \\ ${ }^{1}$ Department of Physics and Astronomy, Stony Brook University, Stony Brook, NY 11794-3800.
}

We review some aspects of spin physics where QCD instantons play an important role. In particular, their large contributions in semi-inclusive deep-inelastic scattering and polarized proton on proton scattering. We also review their possible contribution in the $\mathcal{P}$-odd pion azimuthal charge correlations in peripheral $A A$ scattering at collider energies.

\section{INTRODUCTION}

Dedicated lattice simulations have revealed that the QCD vacuum is characterized by non-trivial topological fluctuations [1]. Instantons and anti-instantons are extrema of the 4-dimensional Euclidean action that carry unit topological charge. They correspond to tunneling between degenerate vacuua. The instanton liquid model with interacting instantons and anti-instantons account for important features of the QCD vacuum, such as the spontaneous breaking of chiral symmetry and the large mass for the $\eta^{\prime}$ meson [4, 5]. It does not account for confinement of static charges. Recently, it was noted that twisted instantons and anti-instantons with finite Polyakov lines preserve most of the features of the instanton liquid model and do account for confinement. QCD instantons may contribute substantially to small angle hadron-hadron scattering [6-10] and possibly gluon saturation at HERA [11, 12, as evidenced by recent lattice investigations [13, 14.

A number of semi-inclusive DIS experiments carried by the CLAS and HERMES collaborations [15 17, and more recently with polarized protons on protons by the STAR and PHENIX collaborations [18 20], have revealed large spin asymmetries in polarized lepton-hadron and hadron-hadron collisions at collider energies. These effects are triggered by $\mathcal{T}$-odd contributions in the scattering amplitude. Perturbative QCD does not support the $\mathcal{T}$-odd contributions, which are usually parametrized in the initial state (Sivers effect) [21, 22] or the final state (Collins effect) [23, 24]. Non-perturbative QCD with instantons allow for large spin asymmetries as discussed by Kochelev and others [25]29]. In 25] a particularly large Pauli form factor was noted, with an important contribution to the Single Spin Asymmetry (SSA) in polarized proton on proton scattering.

In this paper, we review some recent developments regarding our understanding of spin physics in the instanton liquid model. Assuming that the vacuum is populated by semi-classical but interacting instantons and anti-instantons, with the vacuum parameters fixed by the spontaneous breaking of chiral symmetry in bulk, we explicit their effects on semi-inclusive DIS processes as well as singly polarized $p p$ scattering. In both cases, uncorrelated instantons or anti-instantons are at work. We show that the effects of correlations between instantons and anti-instantons through fluctuations are also important in both doubly polarized $p p$ scattering as well as through $\mathcal{P}$-odd effects in peripheral $A A$ scattering. For a complementary and non-instanton based approaches to spin physics, we refer to the recent reviews in [30, 31.

The paper is organized as follows: in section II we detail the role of a single instanton and anti-instanton on the single spin asymmetry in semi-inclusive DIS scattering and in polarized $p p$ scattering. The large instanton contributions appear to be supported by current experiments. In section III we discuss the role of local fluctuations in the compressibility as well as the topological susceptibility in doubly polarized $p p$ and peripheral $A A$ scattering. Our conclusions and prospects follow in section IV. In Appendix A we detail the large instanton vertex contribution to both the electromagnetic and chromo-magnetic interactions with the corresponding large magnetic moments.

\section{INSTANTON LIQUID MODEL}

In this section, we briefly review the instanton liquid model where more details can be found in [4, 32. The discussion of the instanton liquid model is in Euclidean space and our notations are consistent with those in [32. Let us first consider an $S U(2)$ color subgroup gauge field in 4D-Euclidean space. Later we will embed the color subgroup $S U(2)$ in a larger say $S U(3)$ group in our calculation. The Yang-Mills equation of motion reads

$$
D_{\mu} F_{\mu \nu}=0
$$

where

$$
D_{\mu} \equiv \partial_{\mu}+A_{\mu}, \quad \text { and } \quad F_{\mu \nu} \equiv\left[D_{\mu}, D_{\nu}\right]
$$


Eq. (1) is in general involved. However, for (anti-)selfdual field configurations

$$
\widetilde{F}_{\mu \nu}= \pm F_{\mu \nu}
$$

with $\widetilde{F}_{\mu \nu} \equiv \frac{1}{2} \epsilon_{\mu \nu \rho \sigma} F_{\rho \sigma}$, it is easy to show that these configurations naturally satisfy Eq. (1)

$$
D_{\mu} F_{\mu \nu}= \pm D_{\mu} \tilde{F}_{\mu \nu}= \pm \frac{1}{2} \epsilon_{\mu \nu \rho \sigma} D_{\mu} F_{\rho \sigma}=\mp \epsilon_{\mu \nu \rho \sigma} D_{\nu} D_{\rho} D_{\sigma}=0
$$

after using the Bianchi identity. The solution to the (anti-)self-dual equation is referred as an (anti-)instanton solution. Instanton solutions have been found and well studied. For example, the regular one instanton solution and the regular one anti-instanton solution read

$$
\begin{aligned}
& A_{\mu}^{\mathrm{reg}}=-\frac{\bar{\sigma}_{\mu \nu}\left(x-x_{0}\right)^{\nu}}{\left(x-x_{0}\right)^{2}+\rho^{2}}, \quad(k=1, \quad \text { instanton }) \\
& A_{\mu}^{\mathrm{reg}}=-\frac{\sigma_{\mu \nu}\left(x-x_{0}\right)^{\nu}}{\left(x-x_{0}\right)^{2}+\rho^{2}}, \quad(k=-1, \quad \text { anti-instanton }),
\end{aligned}
$$

where

$$
\bar{\sigma}_{\mu \nu} \equiv \frac{1}{2}\left(\bar{\sigma}_{\mu} \sigma_{\nu}-\bar{\sigma}_{\nu} \sigma_{\mu}\right), \quad \text { and } \quad \sigma_{\mu \nu} \equiv \frac{1}{2}\left(\sigma_{\mu} \bar{\sigma}_{\nu}-\sigma_{\nu} \bar{\sigma}_{\mu}\right)
$$

$\sigma_{\mu}=\left(\tau^{a}, i\right), \bar{\sigma}_{\mu}=\left(\tau^{a},-i\right)$ with $\tau^{a}$ pauli matrix. $x_{0}$ is the center of the (anti-)instanton and $\rho$ is the size.

The physical interpretation of an instanton is a tunneling event between degenerate classical vacuua [33] 35 . In this paper, we adopt the instanton liquid model [36], where the QCD vacuum is populated with interacting instantons and anti-instantons with a mean instanton density $n \approx 1 \mathrm{fm}^{-4}$ and an average instanton size of $\rho=1 / 3 \mathrm{fm}$. The classical configurations of instanton and anti-instantons have been observed in lattice simulations using cooling methods. A typical distribution of the topological charge $G \widetilde{G}$ with quantum noise is shown in Fig. 1 (left) and after 25 cooling sweeps in Fig. 1 (right) [3].
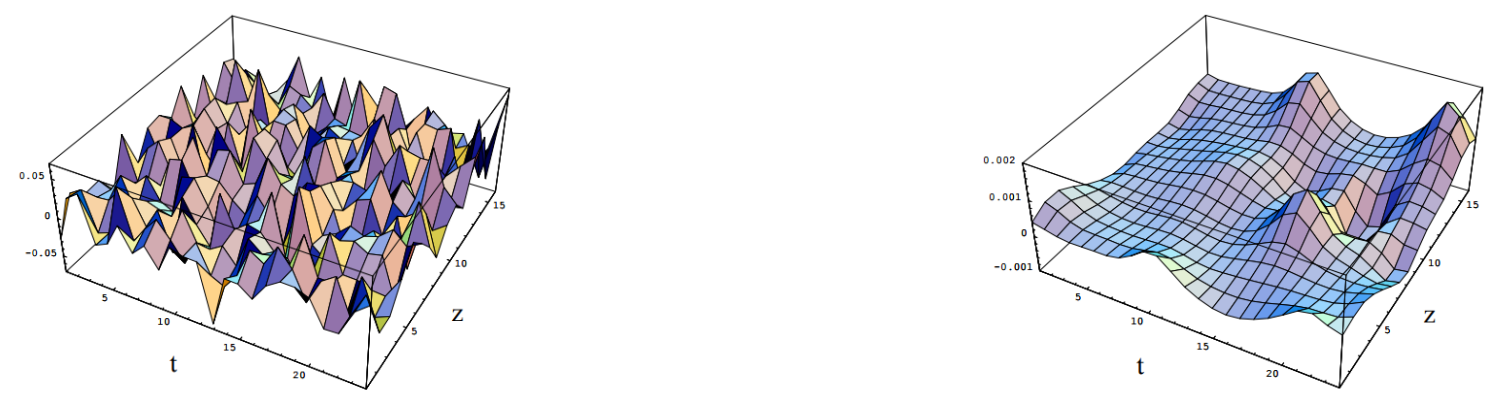

FIG. 1: Left: typical fluctuations of $G \widetilde{G}$ in a lattice simulation with a lattice unit of $0.16 \mathrm{fm}$. Right: the same fluctuations of $G \widetilde{G}$ after 25 cooling sweeps with a lattice unit of $0.14 \mathrm{fm}$.

The instanton ensemble is dilute with a diluteness factor $n \rho^{4} \approx 0.01$. The latter provides a natutal order parameter when evaluating the various contributions in collision processes induced by instantons to be detailed below. Instantons provide an important mechanism for the spontaneous breaking of chiral symmetry and the resolution of the axial $U(1)$ problem through the emergence and delocalization of the light quarks zero modes. Indeed, the massless Dirac equation in instanton background reads $\not D \psi=0$. It is easy to show that in the presence of an instanton 32,

$$
\not D\left(1+\gamma^{5}\right) \psi=0
$$

has no solution while

$$
\not D\left(1-\gamma^{5}\right) \psi=0
$$


has a normalizable left-handed zero mode (regular gauge)

$$
\left(1-\gamma^{5}\right) \psi \sim \bar{\sigma}_{l} \frac{\left(x-x_{0}\right)^{l}}{\left(x-x_{0}\right)^{2}} \frac{\left[\left(x-x_{0}\right)^{2}+\rho^{2}\right]^{\frac{3}{2}}}{\left(x-x_{0}\right)^{2} \rho^{\frac{3}{2}}},
$$

which breaks chiral symmetry. In this paper and other works [27, 28, 37, 42, the calculation is usually implemented in singular gauge, where the instanton configurations are more localized. The transition from regular to singular gauge is defined as

$$
A_{\mu}^{\mathrm{sin}} \equiv U \partial_{\mu} U^{-1}+U A_{\mu}^{\mathrm{reg}} U^{-1}=-\sigma_{\mu \nu} \frac{\left(x-x_{0}\right)^{\nu}}{\left(x-x_{0}\right)^{2}} \frac{\rho^{2}}{\left(x-x_{0}\right)^{2}+\rho^{2}}
$$

where

$$
U=\frac{\left(x-x_{0}\right)_{4}-i\left(x-x_{0}\right)_{k} \sigma_{k}}{\left|x-x_{0}\right|}=-i \sigma^{\mu} \frac{\left(x-x_{0}\right)_{\mu}}{\left|x-x_{0}\right|}
$$

It is readily seen that [37, 39,40

$$
\bar{\sigma}_{\mu}\left(\partial_{\mu}+A_{\mu}^{\sin }\right) \xi=0
$$

with

$$
\xi \sim \sigma_{l} \frac{\left(x-x_{0}\right)_{l}}{\left|x-x_{0}\right|} \frac{\rho^{\frac{3}{2}}}{\left[\left(x-x_{0}\right)^{2}+\rho^{2}\right]^{\frac{3}{2}}}
$$

from which we can construct the zero mode propagator (see App-VIIIA).

A number of important vacuum expectations values, e.g.

$$
\langle\bar{\psi} \psi\rangle, \quad\left\langle\bar{\psi} \gamma^{5} \psi\right\rangle \quad \text { and } \quad\left\langle G_{\mu \nu} G_{\mu \nu}\right\rangle
$$

have been reproduced in the instanton liquid model of the QCD vaccum, in support of the underlying semi-classical gauge and fermionic degrees of freedom. Also many mesonic correlation functions of the type $\langle j(x) j(0)\rangle$, with $j$ a local operator with the quantum numbers of a mesonic state, e.g.

$$
j_{\pi}=\bar{q} \tau^{a} \gamma^{5} q, \quad j_{\rho}=\frac{1}{2} \bar{q} \tau^{a} \gamma_{\mu} q \quad \text { and } \quad j_{\eta}=\bar{q} \gamma^{5} q
$$

have led to detailed agreements with lattice simulations after cooling 3 . These correlation functions are important in understanding the QCD vacuum, hadrons and mesons. In particular, the heavy $\eta^{\prime}$ mass is related to the $U(1)_{A}$ anomaly [4].

\section{SPIN EFFECTS THROUGH ONE INSTANTON}

To best illustrate the important role played by instantons in QCD spin physics, consider a light quark in the fundamental color representation propagating in an external SU(2) colored Yang-Mills gauge field with a chromomagnetic field $\mathbf{B}$ and a chromo-electric field E field. Generically [4]

$$
\left(-\nabla^{2}+4 g_{s} \mathbf{S} \cdot(\mathbf{B} \mp \mathbf{E})\right) \varphi^{ \pm}=0
$$

with $i \nabla=i \partial+A$ and $\mathbf{S}^{a}$ the SU(2) spin generators. The signs in Eq. 16 refer to the chirality of the quark. Large quark amplitudes as polarized zero modes occur when the spin contribution (second term) balances the squared kinetic contribution (first term) in Eq. [16]. For a self-dual instanton with $\mathbf{B}=\mathbf{E}$ the negative chirality quark produces a large zero mode state through the magnetic moment term 


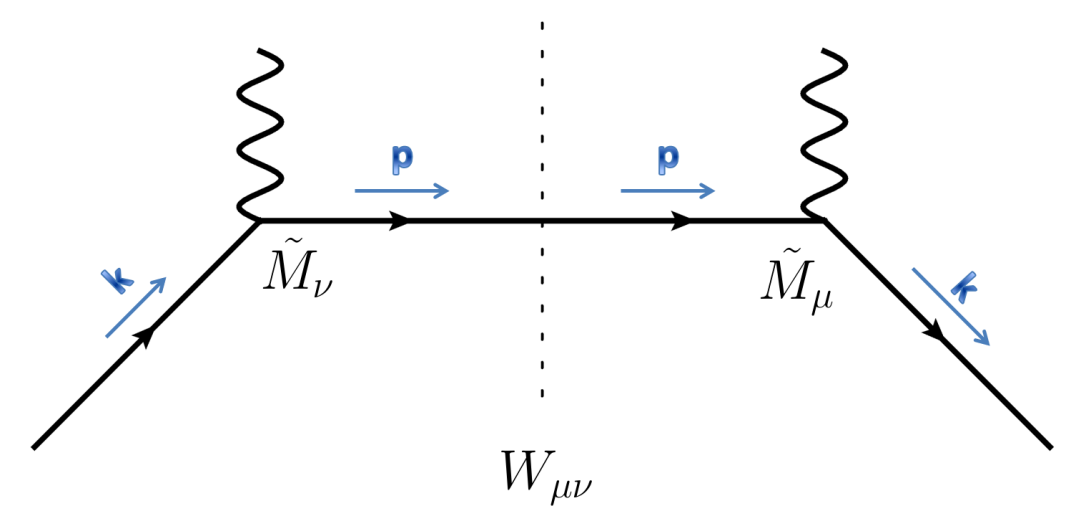

FIG. 2: $\quad p$ and $k$ are the momenta of the incoming and outgoing quark. The lepton and the quark exchange one photon in the single instanton background.

$$
\left(-\nabla^{2}+4 g_{s} \sigma \cdot \mathbf{B}\right) \varphi_{D}^{-}=0
$$

and similarly for an anti-self-dual anti-instanton. Typically $\mathbf{E}, \mathbf{B} \approx 1 / g_{s} \rho^{2}$ with $\rho \approx 1 / 3$ fm the instanton or antiinstanton size in the vacuum and $g_{s}$ the strong gauge coupling. So the induced and large magnetic moment in Eq. (17) is about $\mu_{D} \approx \mathbf{n} \rho^{4}$ where $\mathbf{n} \approx 1 \mathrm{fm}^{-4}$ is the density of instantons in the vacuum [4, 5]. In contrast, perturbative QCD generates small magnetic moments or $\mu_{P T} \approx g_{s}$.

In a similar way, a propagating gluon in an external $\mathrm{SU}(2)$ colored gauge field acquires also an effective and large magnetic moment. Indeed, the analogue of Eq. (16) for a massless gluon in a covariant (Feynman) background gauge $\nabla^{\mu} \mathbf{a}_{\mu}=0$ is

$$
\left(-\nabla^{2} \delta_{\mu \nu}-2 i g_{s} F_{\mu \nu}\right) \mathbf{a}^{\nu}=0
$$

The colored gluon in Eq. (18) has two physical polarizations as both the longitudinal and time-like are gauge artifacts. Using the decomposition $\mathbf{a}_{\mu}=\mathbf{e}_{\mu}^{a} \boldsymbol{\Psi}^{a}$ with $a$ transverse we have

$$
\left(-\nabla^{2}+\frac{g_{s}}{2} \Sigma^{\mu \nu} F_{\mu \nu}\right) \Psi^{a}=0
$$

with $i \Sigma_{\mu \nu}=4 \mathbf{e}_{\mu}^{T} \mathbf{e}_{\nu}$ playing the role of the spin in the gluon transverse polarization space. Eq. (19) is the analogue of Eq. (18) with an induced and large magnetic moment $\mu_{G} \approx \mathbf{n} \rho^{4}$ as well.

In the Appendix we give a quantitative derivation of these estimates. These semi-classical and large spin effects will now be explored in processes with polarized protons and in peripheral $A A$ collisions sensitive to $\mathcal{P}$-odd fluctuations, in the framework of the instanton liquid model.

\section{A. Single Spin Asymmetry in Semi-Inclusive Deep Inelastic Scattering}

To set up the notations for the semi-inclusive processes in deep inelastic scattering, we consider a proton at rest in the LAB frame with transverse polarization as depicted in Fig. 2. The incoming and outgoing leptons are unpolarized. The polarization of the target proton in relation to the DIS kinematics is shown in Fig. 3. Throughout, the spin dependent asymmetries will be evaluated at the partonic level. Their conversion to the hadronic level will follow the qualitative arguments presented in [25 27].

In general, the spin averaged leptonic tensor reads

$$
L^{\mu \nu}=\frac{1}{2} \operatorname{tr}\left[\ell^{\prime} \gamma_{\mu} l \gamma_{\nu}\right]
$$

while the color averaged hadronic tensor in the one instanton background reads 


$$
W_{\mu \nu}=\sum_{\text {color }} \frac{1}{2} \operatorname{tr}\left[\not k \tilde{M}_{\mu} \not p\left(1+\gamma_{5} \phi\right) \gamma_{0}\left(\tilde{M}_{\nu}\right)^{\dagger} \gamma_{0}\right]
$$

with the constituent vertex

$$
\tilde{M}_{\mu}=\gamma_{\mu}+\tilde{M}_{\mu}^{(1)}
$$

that includes both the perturbative $\gamma_{\mu}$ and the non-perturbative insertion $M_{\mu}^{(1)}$. In the Appendix we detail its derivation following the original arguments in [27, 28, 39]. After color averaging, the result is

$$
\operatorname{Im}\left(\left\langle\tilde{M}_{\mu}^{(1)}\right\rangle\right)=-\frac{4 \pi^{2} \rho^{2}}{\lambda_{*} Q^{2}}\left[\gamma_{\mu} \not k+\not p \gamma_{\mu}\right](1-f(\rho Q))
$$

with $f(a)=a K_{1}(a)$ with $K_{1}$ a modified Bessel function. Here $\rho$ is the instanton size and $\lambda_{*} \approx 1 /(0.2 \mathrm{GeV})^{3}[4$, 5 ] is a typical near-mode in the zero-mode-zone as discussed in the Appendix. The electromagnetic gauge invariance in the hadronic tensor in the random instanton model of the vacuum is detailed in [38. The normalized lepton-hadron cross section of Fig 2 follows in the form

$$
\frac{d \sigma}{d x d y d z d \phi}=y \frac{\alpha^{2}}{Q^{6}} L^{\mu \nu} W_{\mu \nu} \sum_{i} e_{i}^{2} f_{i}\left(x, Q^{2}\right) D_{i}(z)
$$

with $y=P \cdot q / P \cdot l$, where $e_{i}$ is the i-parton electric charge, $f_{i}$ its momentum fraction distribution and $D_{i}$ its framentation function.

The perturbative contribution to the hadronic tensor follows from $M_{\mu} \rightarrow \gamma_{\mu}$,

$$
W_{\mu \nu}^{(0)}=\frac{N_{c}}{2} \operatorname{tr}\left[k \gamma_{\mu} \not p \gamma_{\nu}\right]
$$

Thus the leading perturbative contribution

$$
\frac{d^{(0)} \sigma}{d x d y d z d \phi}=2 N_{c} \frac{\alpha^{2}}{Q^{2}} \frac{1+(1-y)^{2}}{y} \sum_{i} e_{i}^{2} f_{i}\left(x, Q^{2}\right) D_{i}(z)
$$

with $N_{c}$ the number of colors. The sum is over the charges $e_{i}$ of the quarks. The non-perturbative instanton contribution to Eq. (24) is a cross contribution in the hadronic tensor in Eq. (24) after inserting the one-instanton vertex Eq. (23)

$$
\begin{aligned}
\left\langle W_{\mu \nu}^{(1)}\right\rangle & =i \frac{2 \pi^{2} \rho^{2}}{\lambda_{*} Q^{2}}[1-f(\rho Q)]\left(\operatorname{tr}\left[\not k \gamma_{\mu} \not p \gamma_{5} \phi\left(\gamma_{\nu} \not p+\not k \gamma_{\nu}\right)\right]-\operatorname{tr}\left[\not k\left(\gamma_{\mu} \not k+\not p \gamma_{\mu}\right) \not p \gamma_{5} \phi \gamma_{\nu}\right]\right) \\
& =-\frac{16 \pi^{2} \rho^{2}}{\lambda_{*} Q^{2}}(1-f(\rho Q))(p+k)_{\{\mu} \epsilon_{\nu\} a b c} s^{a} k^{b} p^{c}
\end{aligned}
$$

where $p \cdot s=0$ and the short notation $(\cdots)_{\{\mu} \epsilon_{\nu\} a b c} \equiv(\cdots)_{\mu} \epsilon_{\nu a b c}+(\cdots)_{\nu} \epsilon_{\mu a b c}$ is used. If we set $p=x P$ and $k=K / z$ and note that $p+k=2 p+q$, then Eq. 27) simplifies

$$
\left\langle W_{\mu \nu}^{(1)}\right\rangle=-\frac{2^{5} \pi^{2} \rho^{2}}{\lambda_{*} Q^{2}} \frac{x^{2}}{z}(1-f(\rho Q))\left(P+\frac{q}{2 x}\right)_{\{\mu} \epsilon_{\nu\} a b c} s^{a} K^{b} P^{c}
$$

Combining Eq. 20 and Eq. 28 yields

$$
\left\langle W_{\mu \nu}^{(1)} L^{\mu \nu}\right\rangle=-\frac{2^{7} \pi^{2} \rho^{2}}{\lambda_{*} Q^{2}} \frac{x^{2}}{z}(1-f(\rho Q)) M\left(E \epsilon_{\nu a b c} l^{\prime \nu} s^{a} K^{b} P^{c}+E^{\prime} \epsilon_{\nu a b c} l^{\nu} s^{a} K^{b} P^{c}\right)
$$


where $E\left(E^{\prime}\right)$ is the energy of the incoming (outgoing) (anti)electron. The leading instanton contribution to the total cross section Eq. (24) can be obtained by inserting Eq. 29) into Eq. (25). The result is

$$
\frac{d^{(1)} \sigma}{d x d y d z d \phi}=\frac{\alpha^{2}}{y Q^{2}} \sum_{q} \Delta_{\perp} q_{q} \frac{64 \pi^{2} \rho^{2} e_{q}^{2} D_{q}(z)}{\lambda_{*} Q} \frac{K_{\perp}}{z Q}(1-f(\rho Q)) \frac{1-y-x^{2} y^{2} \frac{M^{2}}{Q^{2}}}{\sqrt{1+4 \frac{M^{2}}{Q^{2}} x^{2}}} \sin \left(\phi-\phi_{s}\right)
$$

where $\Delta_{\perp} q_{q}\left(x, Q^{2}\right)=s_{\perp} f_{q}(x)$ is the spin polarized distribution function for the quark in the transversely polarized proton. To compare with experiment and for simplicity, we will use the spin structure function [44]

$$
g_{1}\left(x, Q^{2}\right)=\frac{1}{2} \sum_{q} e_{q}^{2}\left(\Delta q_{q}\left(x, Q^{2}\right)+\Delta \bar{q}_{q}\left(x, Q^{2}\right)\right)
$$

where $g_{1}\left(x, Q^{2}\right)$ is the spin distribution function for a longitudinally polarized proton. In other words, we will assume for the sake of an estimate, that the proton has a similar spin distribution function whether polarized longitudinally, or transversely. As we note later, this assumption can be relaxed by calculating the specific instanton contributions to SIDID, $p_{\uparrow} p$ collisions etc. and then using the results to extract the spin polarization distribution from transversely polarized protons. However, this work is outside the scope of this work. Finally, as we are only interested in the SSA in hard scattering processes, we set $D_{q}(z)=1$.

With this in mind, we now normalize Eq. 30 by dividing it by the regular zeroth order differential cross section. The result is

$$
\frac{d^{(1)} \sigma}{d^{(0)} \sigma}=A_{U T}^{\sin \left(\phi-\phi_{s}\right)} \sin \left(\phi-\phi_{s}\right)
$$

where

$$
A_{U T}^{\sin \left(\phi-\phi_{s}\right)}=\frac{32 \pi^{2} \rho^{2}}{N_{c} Q \lambda_{*}}(1-f(\rho Q)) \frac{K_{\perp}}{z Q} \frac{1}{1+(1-y)^{2}} \frac{1-y-x^{2} y^{2} \frac{M^{2}}{Q^{2}}}{\sqrt{1+4 \frac{M^{2}}{Q^{2}} x^{2}}} \frac{g_{1}}{F_{1}}
$$

with $F_{1}(x)=\frac{1}{2} \sum_{q} e_{q}^{2} f_{q}(x)$. In Fig. 4 we compare Eq. (33) to the results reported by HERMES [16. The mean quark zero mode virtuality $\bar{\lambda}$ is tied to the light quark condensate $\chi_{u u}$ through $\bar{\lambda}=1 / \chi_{u u}$. For two flavors we have $\chi_{u u} \approx(200 \mathrm{MeV})^{3}$. The average instanton size in the instanton liquid is $\rho_{*} \approx 1 / 3 \mathrm{fm}$ (upper edge of the band). For comparison, we also show our results for smaller size instantons with $\rho \approx 0.3 \mathrm{fm}$ (lower edge of the band). A better analysis could be done by averaging Eq. (33) over the instanton size distribution as measured say on the lattice. Overall, the instanton contribution through Eq. (33) appears to contribute sizably with the right magnitude and overall behavior. They should be considered in any analysis of the transversely polarized spin asymmetry, along with standard perturbative contributions.

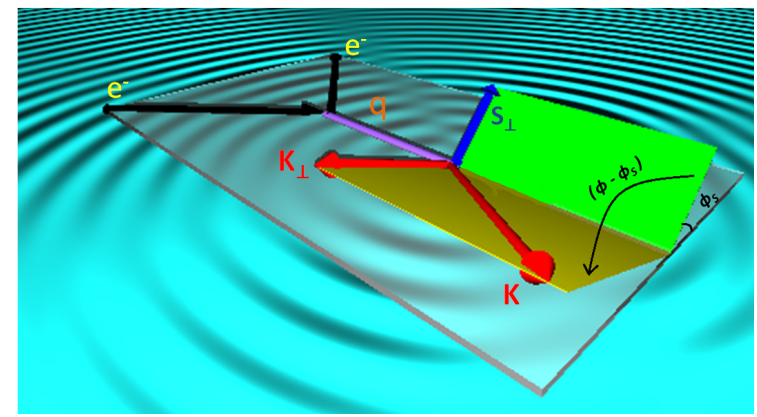

FIG. 3: The lepton and photon are in the same plane. The angle between the transversely polarized spin $s_{\perp}$ and this plane is $\phi_{s}$. The angle between the transversely spatial momentum $K_{\perp}$ of the outgoing pion and the plane is $\phi$. 


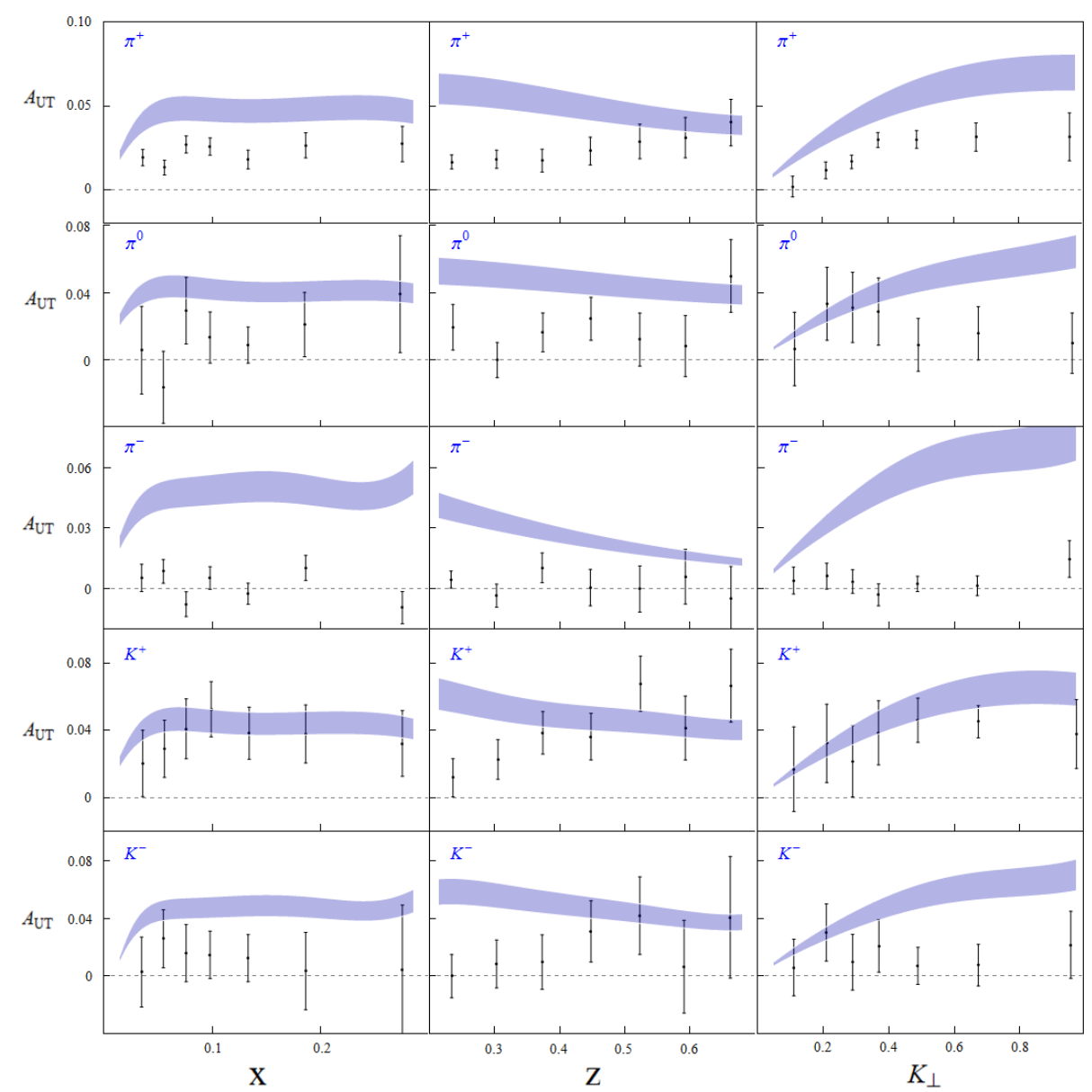

FIG. 4: Transversly polarized spin asymmetry (solid line) versus data [16].

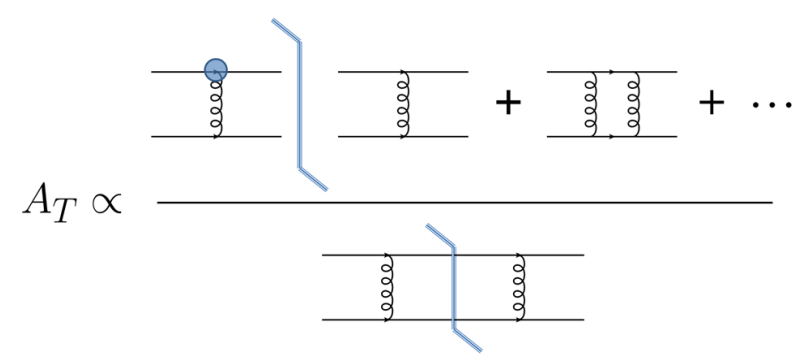

FIG. 5: Schematically diagrammatic contributions to the SSA through the Pauli Form factor [29]

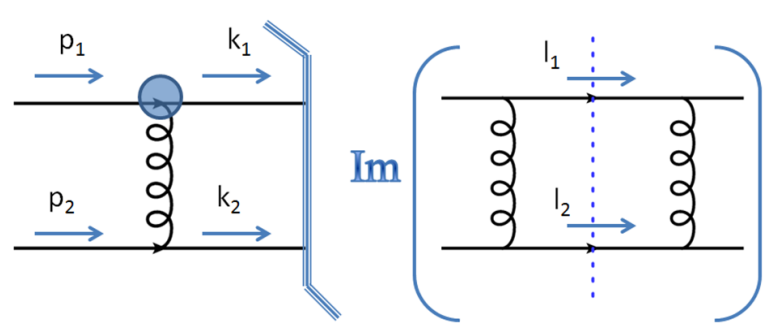

FIG. 6: Leading diagrammatic contribution to the SSA through the Pauli form factor.

\section{B. Single Spin Asymmetry in $p p$ - Transverse parton distribution function}

In this section we briefly review the SSA in semi-inclusive and polarized $p_{\uparrow} p \rightarrow \pi^{ \pm, 0} X$ experiments, following the recent analysis in 29, 41. In going through an instanton, the chirality of the light quark can be flipped as we noted in Eq. 17). Using the Pauli form factor discussed in the Appendix, the SSA follows from the diagrams of Fig. 5 . As noted in 29], the leading diagram contributing to the SSA is displayed in Fig. 6. Note that Fig. 6 is of the same order in $g_{s}$ as the zeroth order diagram in Fig. 5. since the chirality-flip effective vertex Eq. (104) is semi-classical and of order $1 / g_{s}^{2}$. The zeroth order differential cross section reads

$$
d^{(0)} \sigma \sim \frac{64 g_{s}^{4}}{\left|p_{1}-k_{1}\right|^{4}}\left[\left(k_{1} \cdot p_{2}\right)\left(k_{2} \cdot p_{1}\right)+\left(k_{1} \cdot k_{2}\right)\left(p_{1} \cdot p_{2}\right)\right]
$$


The first order differential cross section for the chirality flip reads 45]

$$
d^{(1)} \sigma \sim i \frac{g_{s}^{6}}{\left(k_{1}-p_{1}\right)^{2}} \frac{1}{16 \pi} \frac{(4 \pi)^{\epsilon}}{\Gamma(1-\epsilon)} \frac{\mu^{2 \epsilon}}{s^{\epsilon}} \int_{0}^{1} d y[y(1-y)]^{-\epsilon} \int_{0}^{2 \pi} \frac{d \phi_{l}}{2 \pi} \frac{1}{\left(l_{1}-k_{1}\right)^{2}} \frac{1}{\left(p_{1}-l_{1}\right)^{2}} \mathcal{G}(\Omega)
$$

where $y=\left(1+\cos \theta_{l}\right) / 2, \pm \theta_{l}$ is the longitudinal angle of $l_{1 / 2}$ and

$$
\mathcal{G}(\Omega) \equiv \operatorname{tr}\left[\left(M_{\mu}^{a}\right)^{(1)} \not p_{1} \gamma_{5} \phi \gamma_{\nu} t^{b} l_{1} \gamma_{\rho} t^{c} \not k_{1}\right] \operatorname{tr}\left[\gamma^{\mu} t_{a} \not p_{2} \gamma^{\nu} t_{b} \not_{2} \gamma^{\rho} t_{c} \not k_{2}\right]
$$

From Sec-VIIIB in the Appendix we have

$$
\left\langle\left(M_{\mu}^{a}\right)^{(1)}\right\rangle=-t^{a} \sigma_{\mu \nu} q^{\nu} \Psi
$$

where

$$
\Psi=\frac{F_{g}\left(\rho_{c} Q\right) \pi^{4}\left(n_{I} \rho_{c}^{4}\right)}{m_{q}^{*} g_{s}^{2}}
$$

To simplify the analysis and compare to the existing semi-inclusive data, we use the kinematics

$$
\begin{aligned}
p_{1 / 2} & =\frac{\sqrt{\tilde{s}}}{2}(1,0,0, \pm 1) \\
k_{1 / 2} & =\frac{\sqrt{\tilde{s}}}{2}(1, \pm \sin \theta \sin \phi, \pm \sin \theta \cos \phi, \pm \cos \theta) \\
s & =\left(0,0, s^{\perp}, 0\right)
\end{aligned}
$$

where $\sqrt{\tilde{s}}$ is the total energy of the colliding "partons". It is simple to show that $d^{(1)} \sigma \sim \vec{k}_{1} \cdot\left(\vec{p}_{1} \times \vec{s}\right) \sim \sqrt{\tilde{s}} s^{\perp} k_{1}^{\perp} \sin \phi$, which results in SSA. For simplicity, we calculate the first differential cross section $d^{(1)} \sigma$ with $\phi=\pi / 2$, where the transverse momentum of the outgoing particle lines along the $x$ axis. Straightforward algebra yields

$$
\begin{aligned}
\left\langle d^{(1)}\right\rangle \sigma \sim & s^{\perp} k_{1}^{\perp} \frac{2 g_{s}^{4}}{3 \pi} \frac{\Gamma(-\epsilon)}{\Gamma(2-2 \epsilon) \Gamma(1-\epsilon)} \csc ^{2}(\theta)(4 \pi)^{\epsilon} \frac{\mu^{2 \epsilon}}{s^{\epsilon}}\left(\Psi g_{s}^{2}\right) \\
& \times\left[25 \epsilon-12+\cos \theta(\epsilon(9+2 \epsilon)-4)_{2} F_{1}\left(1,1-\epsilon, 1-2 \epsilon, \sec ^{2} \frac{\theta}{2}\right)+\epsilon(1-\cos \theta)_{2} F_{1}\left(2,1-\epsilon, 1-2 \epsilon, \sec ^{2} \frac{\theta}{2}\right)\right]
\end{aligned}
$$

where ${ }_{2} F(a, b, c ; y)$ is a hypergeometric function. We note that $\left|{ }_{2} F_{1}(1,1,1 ; y)\right|$ is much larger than $\left|{ }_{2} F_{1}^{(0,1,0,0)}(1,1,1 ; y)\right|$ and $\left|{ }_{2} F_{1}^{(0,0,1,0)}(1,1,1 ; y)\right|$ for $y \sim 1$. Therefore

$$
\left\langle d^{(1)} \sigma\right\rangle \sim s^{\perp} k_{1}^{\perp} \frac{2 g_{s}^{4}}{3 \pi}\left(\Psi g_{s}^{2}\right) \csc ^{4}\left(\frac{\theta}{2}\right)(3+\cos \theta)\left(-\frac{1}{\epsilon}+2 \gamma_{\mathrm{E}}+\ln \left(\frac{\tilde{s}}{4 \pi \mu^{2}}\right)\right)
$$

The divergence in Eq. (41) stems from the exchange of soft gluons in the box diagram. In [29] it was regulated using a constituent gluon mass $m_{g}$. For $\theta_{l} \sim 0, \vec{l}_{1}$ is parallel to $\vec{p}_{1}$, and this collinear divergence could be regulated by restricting $-\left(l_{1}-p_{1}\right)^{2}>m_{g}^{2}$ or equivalently setting $y_{\max } \sim 1-c m_{g}^{2} / \tilde{s}$ with $c$ an arbitrary constant of order 1 . This regularization amounts to the substitution

$$
\int_{0}^{1} d y \longrightarrow\left(\int_{0}^{\frac{1+\cos \theta}{2}-c \frac{m_{g}^{2}}{\tilde{s}}}+\int_{\frac{1+\cos \theta}{2}+c \frac{m_{g}^{2}}{\hat{s}}}^{1-c \frac{m_{g}^{2}}{\hat{s}}}\right) d y
$$

in Eq. (35), where we have also regulated the collinear divergence when $\vec{l}_{1}$ is parallel to $\vec{k}_{1}$. Thus

$$
\left(-\frac{1}{\epsilon}+2 \gamma_{\mathrm{E}}+\ln \left(\frac{\tilde{s}}{4 \pi \mu^{2}}\right)\right) \longrightarrow \ln \left(c \frac{\tilde{s}}{m_{g}^{2}}\right)+\ln \left(\frac{1-\cos \theta}{1+\cos \theta}\right)
$$


The regulated SSA is now given by

$$
A_{T}^{\sin \phi} \approx\left\langle\frac{d^{(1)} \sigma}{d^{(0)} \sigma}\right\rangle=s^{\perp} k_{1}^{\perp}\left(\frac{\Psi g_{s}^{2}}{\pi}\right) \frac{(3+\cos \theta)}{6\left(5+2 \cos \theta+\cos ^{2} \theta\right)}\left[\ln \left(c \frac{\tilde{s}}{m_{g}^{2}}\right)+\ln \left(\frac{1-\cos \theta}{1+\cos \theta}\right)\right]
$$

where the zeroth order cross section in Eq. (34) is used for normalization. We note that the single spin asymmetry $A_{T}$ is sometimes referred to as $A_{N}$ in other studies.

To compare with the semi-inclusive data on $p_{\uparrow} p \rightarrow \pi X$, we set $s^{\perp} u\left(x, Q^{2}\right)=\Delta_{s} u\left(x, Q^{2}\right)$ and $s^{\perp} d\left(x, Q^{2}\right)=$ $\Delta_{s} d\left(x, Q^{2}\right)$, with $\Delta_{s} u\left(x, Q^{2}\right)$ and $\Delta_{s} d\left(x, Q^{2}\right)$ as the spin polarized distribution functions of the valence up-quarks and valence down-quarks in the proton respectively. For forward $\pi^{+}, \pi^{-}$and $\pi^{0}$ productions, the SSAs are

$$
\begin{gathered}
A_{T}^{\sin \phi}\left(\pi^{+}\right)=k^{\perp} \frac{\Delta_{s} u\left(x_{1}, Q^{2}\right)}{u\left(x_{1}, Q^{2}\right)}\left(\frac{\Psi g_{s}^{2}}{\pi}\right) \frac{(3+\cos \theta)}{6\left(5+2 \cos \theta+\cos ^{2} \theta\right)}\left[\ln \left(c \frac{\tilde{s}}{m_{g}^{2}}\right)+\ln \left(\frac{1-\cos \theta}{1+\cos \theta}\right)\right] \\
A_{T}^{\sin \phi}\left(\pi^{-}\right)=k^{\perp} \frac{\Delta_{s} d\left(x_{1}, Q^{2}\right)}{d\left(x_{1}, Q^{2}\right)}\left(\frac{\Psi g_{s}^{2}}{\pi}\right) \frac{(3+\cos \theta)}{6\left(5+2 \cos \theta+\cos ^{2} \theta\right)}\left[\ln \left(c \frac{\tilde{s}}{m_{g}^{2}}\right)+\ln \left(\frac{1-\cos \theta}{1+\cos \theta}\right)\right] \\
A_{T}^{\sin \phi}\left(\pi^{0}\right)=k^{\perp} \frac{\Delta_{s} u\left(x_{1}, Q^{2}\right)+\Delta_{s} d\left(x_{1}, Q^{2}\right)}{u\left(x_{1}, Q^{2}\right)+d\left(x_{1}, Q^{2}\right)}\left(\frac{\Psi g_{s}^{2}}{\pi}\right) \frac{(3+\cos \theta)}{6\left(5+2 \cos \theta+\cos ^{2} \theta\right)}\left[\ln \left(c \frac{\tilde{s}}{m_{g}^{2}}\right)+\ln \left(\frac{1-\cos \theta}{1+\cos \theta}\right)\right]
\end{gathered}
$$

According to [46, 47]

$$
\begin{aligned}
\frac{\Delta_{s} u\left(x, Q^{2}\right)}{u\left(x, Q^{2}\right)} & =0.959-0.588\left(1-x^{1.048}\right) \\
\frac{\Delta_{s} d\left(x, Q^{2}\right)}{d\left(x, Q^{2}\right)} & =-0.773+0.478\left(1-x^{1.243}\right) \\
\frac{u\left(x, Q^{2}\right)}{d\left(x, Q^{2}\right)} & =0.624(1-x)
\end{aligned}
$$

These results can be compared to the experimental measurements in [48. For simplicity, we assume the same fraction for each proton $\left\langle x_{1}\right\rangle=\left\langle x_{2}\right\rangle=\langle x\rangle$. This assumption is revisited in Appendix C. We set $\left\langle k^{\perp}\right\rangle \approx\left\langle K_{\perp}\right\rangle$ as the transverse momentum of the outgoing pion. We then have $\sqrt{s}\langle x\rangle\langle\sin \theta\rangle=2\left\langle K_{\perp}\right\rangle$ and $\langle x\rangle\langle\cos \theta\rangle=\left\langle x_{F}\right\rangle$. For large $\sqrt{s}$, we also have $\langle Q\rangle \approx\left\langle K_{\perp}\right\rangle \sqrt{\langle x\rangle /\left\langle x_{F}\right\rangle}$. We set $c=2$ and $\left\langle K_{\perp}\right\rangle=2 \mathrm{GeV}$ for the outgoing pions. $n_{I} \approx 1 / \mathrm{fm}^{4}$ is the effective instanton density, $\rho \approx 1 / 3 \mathrm{fm}$ the typical instanton size and $m_{q}^{*} \approx 300 \mathrm{MeV}$ the constitutive quark mass in the instanton vacuum. $m_{g} \approx 420 \mathrm{MeV}$ is the effective gluon mass in the instanton vacuum[49]. In Fig. 7] (left) we display the results Eq. (45)-Eq. (47) as a function of the parton fraction $x_{F}$ for both the charged and uncharged pions at $\sqrt{s}=19.4 \mathrm{GeV}$ [48]. Fig. 7 (right) is similar to (left) except for the fact that the divergence in Eq. (35) is now regulated by using a constituent gluon mass as in [29]. Again, we find that the instanton contribution is large with the right order of magnitude and trend. This contribution may not be complete but should be added to current perturbative estimates. Below we show that instantons also give large contributions to the Double Spin Asymmetry (DSA), in particular to DSA at $\sqrt{s}=62.4 \mathrm{GeV}$ which is to be compared with RHIC's future measurement (see Sec-IV A). Finally, we also compare our results with the data in Fig. 8 (left), which is from [50, and the data (right), which is from [51]. In sum, the anomalous Pauli form factor can reproduce the correct magnitude of the observed SSA in polarized $p_{\uparrow} p \rightarrow \pi X$ for reasonable vacuum parameters.

\section{SPIN EFFECTS THROUGH TWO INSTANTONS}

\section{A. Double Spin Asymmetry in $p p$}

The same Pauli form factor and vacuum parameters can be used to assess the role of the QCD instantons on doubly polarized and semi-inclusive $p_{\uparrow} p_{\uparrow} \rightarrow \pi \pi X$ processes. The Double Spin Asymmetry (DSA) is defined as

$$
A_{\mathrm{DS}}=\frac{\sigma^{\uparrow \uparrow+\downarrow \downarrow}-\sigma^{\downarrow \uparrow+\uparrow \downarrow}}{\sigma^{\uparrow \uparrow+\downarrow \downarrow}+\sigma^{\downarrow \uparrow+\uparrow \downarrow}}
$$



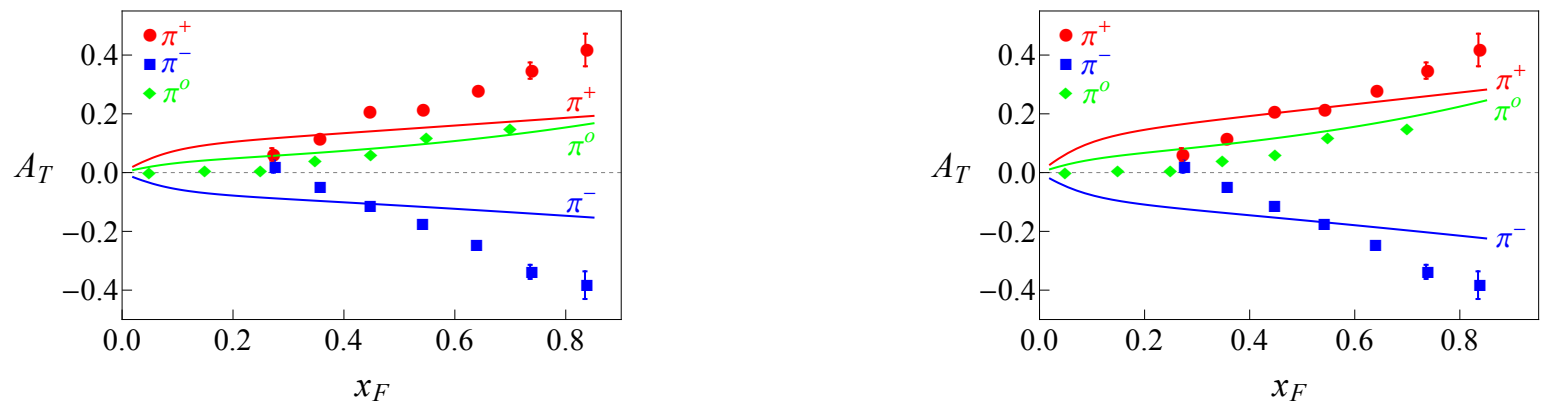

FIG. 7: $x_{F}$ dependent SSA in $p_{\uparrow} p \rightarrow \pi X$ collisions at $\sqrt{s}=19.4 \mathrm{GeV}$ [8]. The solid lines are the analytical results in Eq. 45]Eq. (47) with $c=2$ (left). A regulator using a massive gluon propagator yields the results (right).
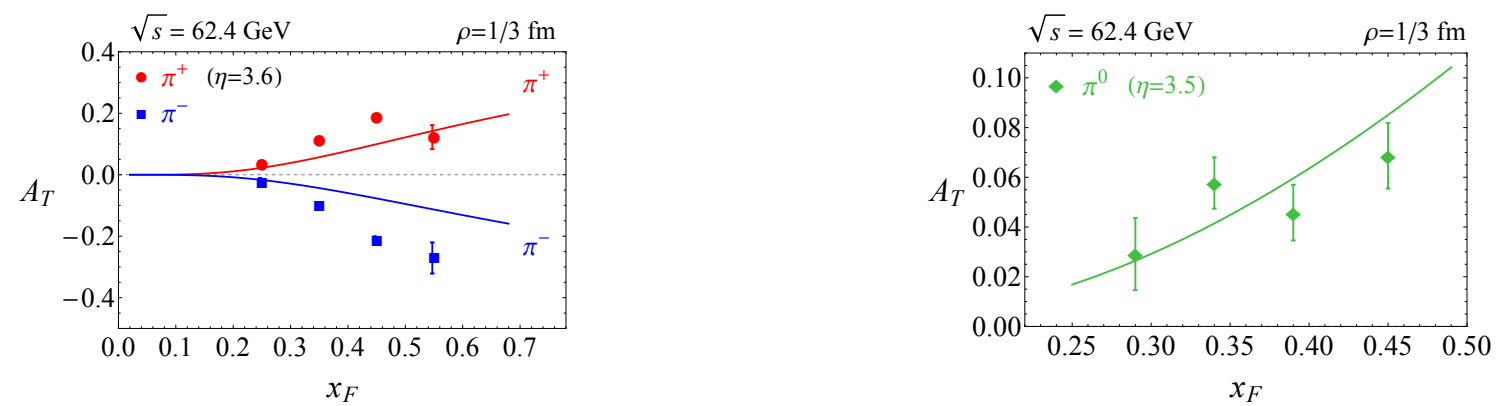

FIG. 8: $x_{F}$ dependent SSA in $p_{\uparrow} p \rightarrow \pi X$ collisions at $\sqrt{s}=62.4 \mathrm{GeV}$. Data (left) is from [50. Data (right) is from [51].

with the proton beam polarized along the transverse direction. The valence quark from the polarized proton $P_{1}$ exchanges one gluon with the valence quark from the polarized proton $P_{2}$ as shown in Fig. 9. At large $\sqrt{s}$, Fig. 9. (a) is dominant in forward pion production and Fig. 9-(b) is dominant in backward pion production. For Fig. 9f(a), the differential cross section reads

$$
d \sigma \sim \frac{g_{s}^{4}}{\left|p_{1}-k_{1}\right|^{4}} \sum_{\text {color }} \operatorname{tr}\left[M_{\mu}^{a} \not p_{1}\left(1+\gamma_{5} \phi_{1}\right) \gamma_{0}\left(M_{\nu}^{b}\right)^{\dagger} \gamma_{0} \not k_{1}\right] \operatorname{tr}\left[M_{\mu}^{a} \not p_{2}\left(1+\gamma_{5} \phi_{2}\right) \gamma_{0}\left(M_{\nu}^{b}\right)^{\dagger} \gamma_{0} \not k_{2}\right]
$$

where $M_{\mu}$ is proportional to $\left(\mathbf{P}_{+}+\mathbf{P}_{-}\right)$as detailed in VIIIB of the Appendix. To second order, we approximately have

$$
\left\langle d^{(2)} \sigma\right\rangle \sim(\cdots)\left\langle\left(\mathbf{P}_{+}+\mathbf{P}_{-}\right)^{2}\right\rangle \approx(\cdots)\left\langle\mathbf{P}_{+}+\mathbf{P}_{-}\right\rangle^{2}
$$

since the instanton liquid is dilute. The contribution to the DSA then follows from simple algebra

$$
\begin{aligned}
\left\langle d^{(2)} \sigma\right\rangle \sim & \frac{256}{\left|p_{1}-k_{1}\right|^{4}}\left(\psi g_{s}^{2}\right)^{2}\left[\left(k_{1} \cdot s_{1}\right)\left(k_{1} \cdot s_{2}\right)\left(k_{2} \cdot p_{1}\right)\left(k_{2} \cdot p_{2}\right)-\left(k_{1} \cdot p_{1}\right)\left(k_{1} \cdot s_{2}\right)\left(k_{2} \cdot p_{2}\right)\left(k_{2} \cdot s_{1}\right)\right. \\
& -\left(k_{1} \cdot s_{1}\right)\left(k_{1} \cdot s_{2}\right)\left(k_{2} \cdot p_{2}\right)\left(p_{1} \cdot p_{2}\right)+\left(k_{1} \cdot k_{2}\right)\left(k_{1} \cdot p_{1}\right)\left(k_{2} \cdot p_{2}\right)\left(s_{1} \cdot s_{2}\right)-\left(k_{1} \cdot p_{1}\right)\left(k_{1} \cdot p_{2}\right)\left(k_{2} \cdot p_{2}\right)\left(s_{1} \cdot s_{2}\right) \\
& -\left(k_{1} \cdot p_{1}\right)\left(k_{2} \cdot p_{1}\right)\left(k_{2} \cdot p_{2}\left(s_{1} \cdot s_{2}\right)+\left(k_{1} \cdot p_{1}\right)\left(k_{2} \cdot p_{2}\right)\left(p_{1} \cdot p_{2}\right)\left(s_{1} \cdot s_{2}\right)-\left(k_{1} \cdot p_{2}\right)\left(k_{1} \cdot s_{1}\right)\left(k_{2} \cdot p_{1}\right)\left(k_{2} \cdot s_{2}\right)\right. \\
& \left.+\left(k_{1} \cdot p_{1}\right)\left(k_{1} \cdot p_{2}\right)\left(k_{2} \cdot s_{1}\right)\left(k_{2} \cdot s_{2}\right)+\left(k_{1} \cdot k_{2}\right)\left(k_{1} \cdot s_{1}\right)\left(k_{2} \cdot s_{2}\right)\left(p_{1} \cdot p_{2}\right)-\left(k_{1} \cdot p_{1}\right)\left(k_{2} \cdot s_{1}\right)\left(k_{2} \cdot s_{2}\right)\left(p_{1} \cdot p(2)\right) \&\right)
\end{aligned}
$$

after using the identity

$$
\begin{aligned}
& \operatorname{tr}\left[\left(\gamma_{\mu} \not \not-\not \phi \gamma_{\mu}\right) \not p \gamma_{5} \phi \gamma_{\nu} \not k\right]+\operatorname{tr}\left[\gamma_{\mu} \not p \gamma_{5} \phi\left(\not \gamma_{\nu}-\gamma_{\nu} \not\right) \not k\right] \\
= & \operatorname{tr}\left[\left(\gamma_{\mu} \not k+\not p \gamma_{\mu}\right) \not p \gamma_{5} \phi \gamma_{\nu} \not k\right]+\operatorname{tr}\left[\gamma_{\mu} \not p \gamma_{5} \phi\left(\not k \gamma_{\nu}+\gamma_{\nu} \not p\right) \not k\right] \\
= & 8 i\left[p_{\mu} \epsilon(\nu, k, p, s)-p_{\nu} \epsilon(\mu, k, p, s)+(k \cdot p) \epsilon(\mu, \nu, k, s)-(k \cdot s) \epsilon(\mu, \nu, k, p)\right]
\end{aligned}
$$

with $q=k-p$ and $p \cdot s=0$ because the protons are transversely polarized. For an empirical application of Eq. (52) we adopt the simple kinematical set up in Eq. (64). Thus 


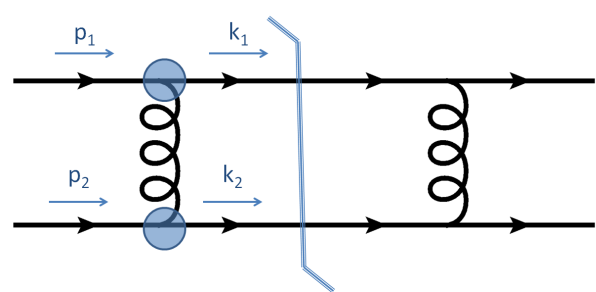

(a)

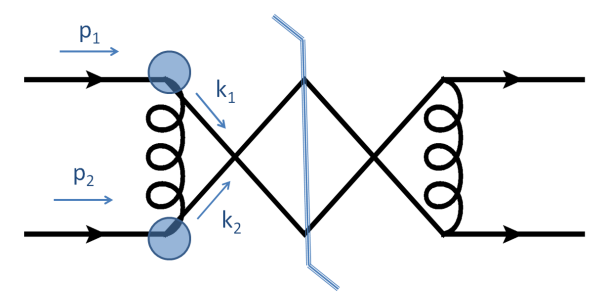

(b)

FIG. 9: The valence quark in polarized proton $p_{1}$ exchange one gluon with the valence quark in the polarized proton $p_{2}$.

$$
\left\langle d^{(2)} \sigma\right\rangle \sim-\frac{4}{\left|p_{1}-k_{1}\right|^{4}}\left(\psi g_{s}^{2}\right)^{2} \tilde{s}^{3} s_{1}^{\perp} s_{2}^{\perp}(1-\cos \theta)^{2}[4+\cos (\theta-2 \phi)+2 \cos (2 \phi)+\cos (\theta+2 \phi)]
$$

After adding the contribution of Fig. 9f(a) and Fig. 9-(b), and averaging over the transverse direction $\phi$, we finally obtain

$$
\frac{d^{(2)} \sigma}{d^{(0)} \sigma} \sim-4 s_{1}^{\perp} s_{2}^{\perp}\left(\frac{\pi^{4} n_{I} \rho_{c}^{4}}{m_{q}^{*} g_{s}^{2}}\right)^{2} \frac{F_{g}^{2}\left[\rho \sqrt{\frac{\tilde{s}(1-\cos \theta)}{2}}\right] \tilde{s}+F_{g}^{2}\left[\rho \sqrt{\frac{\tilde{s}(1+\cos \theta)}{2}}\right] \tilde{s}}{\frac{5+2 \cos \theta+\cos ^{2} \theta}{(1-\cos \theta)^{2}}+\frac{5-2 \cos \theta+\cos ^{2} \theta}{(1+\cos \theta)^{2}}}
$$

Our DSA results can now be compared to future experiments at collider energies. Specifically, our DSA for dijet productions are

$$
\begin{gathered}
A_{\pi^{+} \pi^{+}}=-\frac{1}{8} \frac{\Delta_{s} u\left(x_{1}, Q^{2}\right)}{u\left(x_{1}, Q^{2}\right)} \frac{\Delta_{s} u\left(x_{2}, Q^{2}\right)}{u\left(x_{2}, Q^{2}\right)}\left(\frac{\pi^{3} n_{I} \rho_{c}^{4}}{m_{q}^{*} \alpha_{s}}\right)^{2} \frac{F_{g}^{2}\left[\rho \sqrt{\frac{\tilde{s}(1-\cos \theta)}{2}}\right] \tilde{s}+F_{g}^{2}\left[\rho \sqrt{\frac{\tilde{s}(1+\cos \theta)}{2}}\right] \tilde{s}}{\left(5+10 \cos ^{2} \theta+\cos ^{4} \theta\right) \csc ^{4} \theta} \\
A_{\pi^{-} \pi^{-}}=-\frac{1}{8} \frac{\Delta_{s} d\left(x_{1}, Q^{2}\right)}{d\left(x_{1}, Q^{2}\right)} \frac{\Delta_{s} d\left(x_{2}, Q^{2}\right)}{d\left(x_{2}, Q^{2}\right)}\left(\frac{\pi^{3} n_{I} \rho_{c}^{4}}{m_{q}^{*} \alpha_{s}}\right)^{2} \frac{F_{g}^{2}\left[\rho \sqrt{\frac{\tilde{s}(1-\cos \theta)}{2}}\right] \tilde{s}+F_{g}^{2}\left[\rho \sqrt{\frac{\tilde{s}(1+\cos \theta)}{2}}\right] \tilde{s}}{\left(5+10 \cos ^{2} \theta+\cos ^{4} \theta\right) \csc ^{4} \theta} \\
A_{\pi^{+} \pi^{-}}=-\frac{1}{8} \frac{\Delta_{s} u\left(x_{1}, Q^{2}\right) \Delta_{s} d\left(x_{2}, Q^{2}\right)+\Delta_{s} d\left(x_{1}, Q^{2}\right) \Delta_{s} u\left(x_{2}, Q^{2}\right)}{u\left(x_{1}, Q^{2}\right) d\left(x_{2}, Q^{2}\right)+d\left(x_{1}, Q^{2}\right) u\left(x_{2}, Q^{2}\right)}\left(\frac{\pi^{3} n_{I} \rho_{c}^{4}}{m_{q}^{*} \alpha_{s}}\right)^{2} \\
\times \frac{F_{g}^{2}\left[\rho \sqrt{\frac{\tilde{s}(1-\cos \theta)}{2}}\right] \tilde{s}+F_{g}^{2}\left[\rho \sqrt{\frac{\tilde{s}(1+\cos \theta)}{2}}\right] \tilde{s}}{\left(5+10 \cos ^{2} \theta+\cos ^{4} \theta\right) \csc ^{4} \theta}
\end{gathered}
$$

To compare our calculations with the future measurements at RHIC, we use the same kinematics in Fig. 8 . $\sqrt{s}=$ $62.4 \mathrm{GeV}$ and $\eta=3.5$. The value of $\alpha_{s}$ is from [52. Our estimates for charged di-jet production in semi-inclusive DSA are displayed in Fig. 10.

\section{P-ODD EFFECTS THROUGH INSTANTON FLUCTUATIONS}

It is commonly accepted that in a typical non-central $A u A u$ collision at RHIC as illustrated in Fig. 11 (left), the flying fragments create a large magnetic field that strongly polarizes the wounded or participant nucleons in the final state. The magnetic field is typically $e B / m_{\pi}^{2} \approx 1$ at RHIC and $e B / m_{\pi}^{2} \approx 15$ at the LHC and argued to last for about $1-3 \mathrm{fm} / c$ [53. We recall that in these units $m_{\pi}^{2} \approx 10^{18}$ Gauss which is substantial. As a result, large $\mathcal{P}$-odd pion azimuthal charge correlations were predicted to take place in peripheral heavy ion collisions [54 57].

In this section, we would review the analysis in 42 and show that a large contribution to the $\mathcal{P}$-odd pion azimuthal charge correlations may stem from the prompt part of the collision as each of the incoming nucleus polarizes strongly the participating nucleons from its partner nucleus during the collision process as illustrated in Fig. 11 (right). The magnetic field is strong but short lived in the initial state, lasting for about $1 / 2 \mathrm{fm} / c$ for a typical heavy ion collision at current collider energies. Polarized proton on proton scattering can exhibit large chirality flip effects through instanton and anti-instanton fluctuations as we now show. 


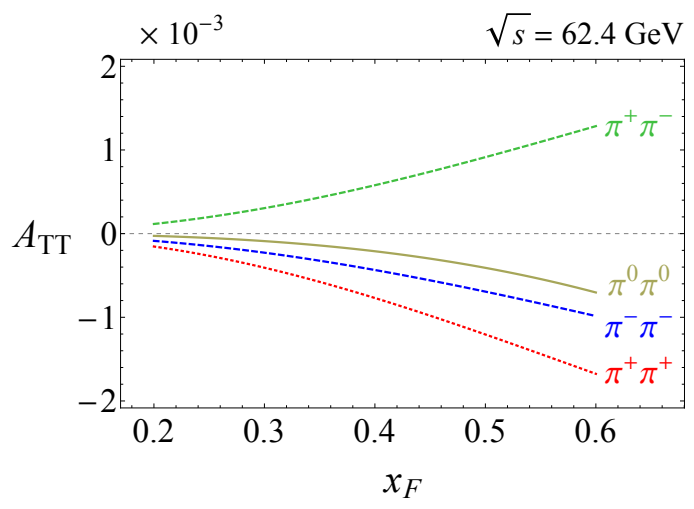

FIG. 10: Predictions for charged di-jet production in semi-inclusive DSA.

\section{A. $\quad \mathcal{P}$-odd Effects in the Instanton Vacuum}
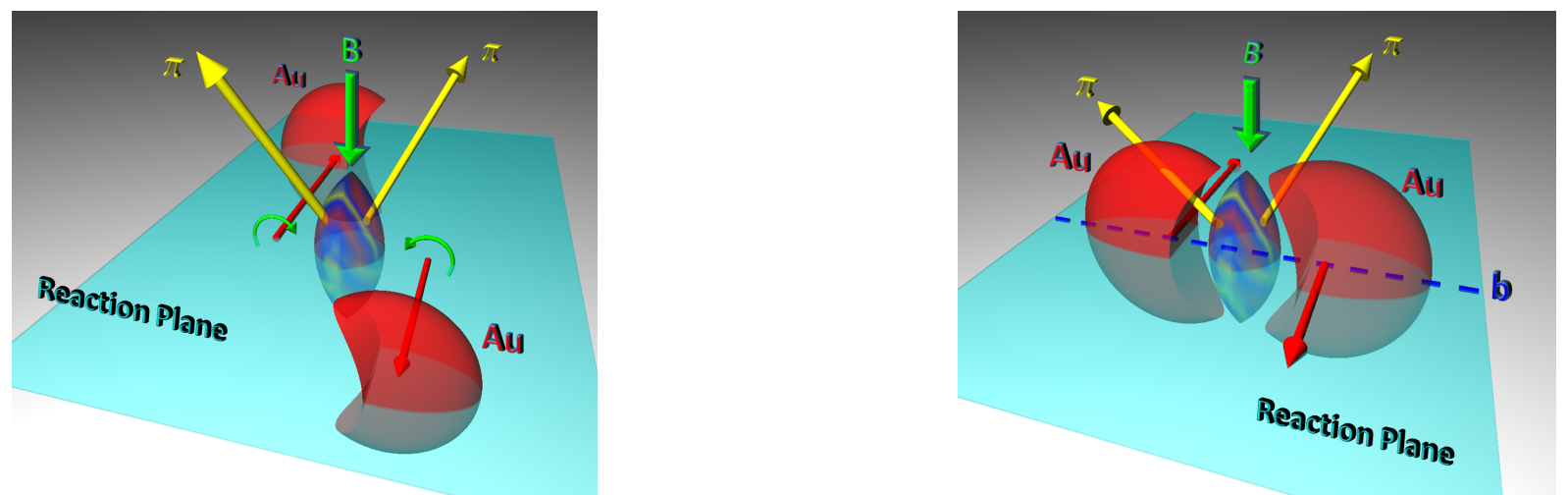

FIG. 11: 2-pion correlations in $A u A u$ after the collision.

Consider the typical parton-parton scattering amplitude of Fig. 12 with 2-gluon exchanges. In each collision, the colliding "parton" $p_{i}$ has spin $s_{i}$, and thus $u\left(p_{i}\right) \bar{u}\left(p_{i}\right)=\frac{1}{2} p_{i}\left(1+\gamma_{5} \phi_{i}\right)$. The parton $p_{1}$ from the A-nucleus encounters an instanton or anti-instanton as depicted by the gluonic form-factor. Rewrite the Eq. (102)

$$
M_{\mu}^{a}=t^{a}\left[\gamma_{\mu}-\mathbf{P}_{+} \gamma_{+} \sigma_{\mu \nu} q^{\nu} \Psi-\mathbf{P}_{-} \gamma_{-} \sigma_{\mu \nu} q^{\nu} \Psi\right]
$$

with $\mathbf{P}_{+}=1$ stands for an instanton insertion and $\mathbf{P}_{-}=1$ for an anti-instanton insertion. In establishing Eq. 102 , the instanton and anti-instanton zero modes are assumed to be undistorted by the prompt external magnetic field. Specifically, the chromo-magnetic field $B_{G}$ is much stronger than the electro-magnetic field $B$, i.e. $\left|g_{s} B_{G}\right| \gg|e B| \approx$ or $m_{\pi}^{2} \rho_{c}^{2} \approx 0.004 \ll 1$. The deformation of the instanton zero-modes by a strong magnetic field have been discussed in [58. They will not be considered here.

In terms of Eq. 102, the contribution of Fig. 12 to the differential cross section is

$$
d \sigma \sim \frac{g_{s}^{4}}{\left|p_{1}-k\right|^{4}} \operatorname{tr}\left[M_{\mu}^{a} \not p_{1}\left(1+\gamma_{5} \phi_{1}\right) \gamma_{0}\left(M_{\nu}^{b}\right)^{\dagger} \gamma_{0} \not k\right] \operatorname{tr}\left[\gamma^{\mu} t_{a} \not p_{2}\left(1+\gamma_{5} \phi_{2}\right) \gamma^{\nu} t_{b} \not k^{\prime}\right]
$$

which can be decomposed into $d \sigma \approx d \sigma^{(0)}+d \sigma^{(1)}$ in the dilute instanton liquid. The zeroth order contribution is

$$
d^{(0)} \sigma \sim \frac{64 g_{s}^{4}}{\left|p_{1}-k\right|^{4}}\left[2\left(k \cdot p_{2}\right)\left(p_{1} \cdot p_{2}\right)+\left(k \cdot p_{1}\right)\left(p_{1} \cdot p_{2}-k \cdot p_{2}\right)\right]
$$

where we used $k^{\prime}=p_{1}+p_{2}-k$. The first order contribution is 


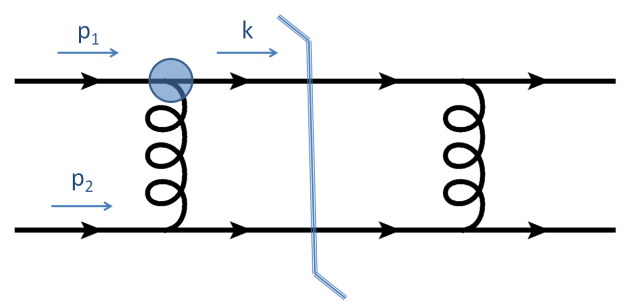

FIG. 12: Gluon Exchange. The blob is an instanton or anti-instanton insertion. See text.

$$
d^{(1)} \sigma \sim \frac{64 g_{s}^{4}}{\left|p_{1}-k\right|^{4}}\left[\left(p_{1} \cdot p_{2}\right)^{2}+\left(k \cdot p_{2}\right)\left(p_{1} \cdot p_{2}\right)\right]\left(k \cdot s_{1}\right) \Psi\left(\mathbf{P}_{+}-\mathbf{P}_{-}\right)
$$

after using $p_{1} \cdot s_{1}=0$ and $p_{1}^{2}=k^{2}=0$. Converting to standard parton kinematics with $p_{1} \rightarrow x_{1} P_{1}, p_{2} \rightarrow x_{2} P_{2}$ and $k \rightarrow K / z$, we obtain for the ratio of the $\mathcal{P}$-odd to $\mathcal{P}$-even contributions in the differential cross section

$$
\frac{d^{(1)} \sigma}{d^{(0)} \sigma}=\frac{x_{1}\left(P_{1} \cdot P_{2}\right)^{2}+\frac{1}{z}\left(K \cdot P_{2}\right)\left(P_{1} \cdot P_{2}\right)}{2\left(K \cdot P_{2}\right)\left(P_{1} \cdot P_{2}\right)+\left(K \cdot P_{1}\right)\left(\frac{x_{1}}{x 2} P_{1} \cdot P_{2}-\frac{K \cdot P_{2}}{z x 2}\right)}\left(K \cdot s_{1}\right) \Psi\left(\mathbf{P}_{+}-\mathbf{P}_{-}\right)
$$

Now consider the kinematics appropriate for the collision set up in Fig. 11 ,

$$
\begin{aligned}
P_{1 / 2} & =\frac{\sqrt{s}}{2}(1,0,0, \pm 1) \\
K & =\left(E, K_{\perp} \cos \Delta \phi, K_{\perp} \sin \Delta \phi, \frac{\sqrt{s}}{2} x_{F}\right) \\
s_{1} & =\left(0,0, s_{1}^{\perp}, 0\right)
\end{aligned}
$$

where $K_{\perp}$ and $E^{2}=K_{\perp}^{2}+s x_{F}^{2} / 4+m_{\pi}^{2}$ are the transverse momentum and total squared energy of the outgoing pion respectively. $x_{F}$ is the pion longitudinal momentum fraction. Thus

$$
\lim _{s \rightarrow \infty} \frac{d^{(1)} \sigma}{d^{(0)} \sigma}=(\sin \Delta \phi) s_{1}^{\perp} \frac{x_{F}+x_{1} z}{x_{F} z} \frac{K_{\perp}}{m_{q}^{*}} \frac{\pi^{3}\left(n_{I} \rho_{c}^{4}\right)}{8 \alpha_{s}} F_{g}\left(\rho \sqrt{\frac{x_{1}}{x_{F} z}\left(K_{\perp}^{2}+m_{\pi}^{2}\right)}\right)\left(\mathbf{P}_{-}-\mathbf{P}_{+}\right)
$$

We note that Eq. (65) vanishes after averaging over the instanton liquid background which is $\mathcal{P}$-even

$$
\left\langle\frac{d^{(1)} \sigma}{d^{(0)} \sigma}\right\rangle=0
$$

since on average $\langle\mathbf{Q}\rangle=\left\langle\mathbf{P}_{+}-\mathbf{P}_{-}\right\rangle=0$.

\section{B. $\quad \mathcal{P}$-odd effects in AA Collisions}

Now consider hard $p p$ collisions in peripheral $A A$ collisions as illustrated in Fig. 11 (right). The Magnetic field is strong enough at the collision to partially polarize the colliding protons. Say $c \%$ of the wounded protons from a given nucleus get polarized by the partner colliding nucleus. For simplicity, we set $s_{\perp} u\left(x, Q^{2}\right)=c \% \Delta_{s} u\left(x, Q^{2}\right)$ and $s_{\perp} d\left(x, Q^{2}\right)=c \% \Delta_{s} d\left(x, Q^{2}\right)$, with $\Delta_{s} u\left(x, Q^{2}\right)$ and $\Delta_{s} d\left(x, Q^{2}\right)$ as the spin polarized distribution functions of the valence up-quarks and valence down-quarks in the proton respectively. We also assume that the outgoing $u$ quark turns to $\pi^{+}$and that the outgoing $d$ quark turns to $\pi^{-}$. With this in mind, we may rewrite the ratio of differential contributions in Eq. (65) following [59]62] as

$$
\frac{d \mathbf{N}}{d \phi_{\alpha}} \sim 1-2 a_{\alpha} \sin \left(\phi-\Psi_{R P}\right)
$$


with $\alpha= \pm$ or

$$
a_{+}=\frac{\Delta_{s} u\left(x, Q^{2}\right)}{u\left(x, Q^{2}\right)} \Upsilon \mathbf{Q} \quad a_{-}=\frac{\Delta_{s} d\left(x, Q^{2}\right)}{d\left(x, Q^{2}\right)} \Upsilon \mathbf{Q}
$$

and

$$
\Upsilon \equiv \frac{x_{F}+x z}{x_{F} z} \frac{K_{\perp}}{m_{q}^{*}} \frac{\pi^{3}\left(n_{I} \rho_{c}^{4}\right)}{16 \alpha_{s}} F_{g}\left(\rho \sqrt{\frac{x}{x_{F} z}\left(K_{\perp}^{2}+m_{\pi}^{2}\right)}\right)
$$

While on average $\left\langle a_{\alpha}\right\rangle=0$ since $\langle\mathbf{Q}\rangle_{V}=0$, in general $\left\langle a_{\alpha} a_{\beta}\right\rangle \neq 0$ for the 2-particle correlations. Explicitly

$$
\begin{aligned}
& -\left\langle a_{\pi^{+}} a_{\pi^{-}}\right\rangle=-\left(\frac{\Delta_{s} u\left(x, Q^{2}\right)}{u\left(x, Q^{2}\right)} \frac{\Delta_{s} d\left(x, Q^{2}\right)}{d\left(x, Q^{2}\right)}\right) \Upsilon^{2}\left\langle\mathbf{Q}^{2}\right\rangle_{V} \\
& -\left\langle a_{\pi^{+}} a_{\pi^{+}}\right\rangle=-\left(\frac{\Delta_{s} u\left(x, Q^{2}\right)}{u\left(x, Q^{2}\right)}\right)^{2} \Upsilon^{2}\left\langle\mathbf{Q}^{2}\right\rangle_{V} \\
& -\left\langle a_{\pi^{-}} a_{\pi^{-}}\right\rangle=-\left(\frac{\Delta_{s} d\left(x, Q^{2}\right)}{d\left(x, Q^{2}\right)}\right)^{2} \Upsilon^{2}\left\langle\mathbf{Q}^{2}\right\rangle_{V}
\end{aligned}
$$

For reasonable values of $\langle x\rangle,\left\langle a_{\pi^{+}} a_{\pi^{+}}\right\rangle \sim\left\langle a_{\pi^{-}} a_{\pi^{-}}\right\rangle \sim-\left\langle a_{\pi^{+}} a_{\pi^{-}}\right\rangle$as expected [59 62].

A more quantitative comparison to the reported data in [59, 62] can be carried out by estimating the fluctuations of the topological charge $\mathbf{Q}$ in the prompt collision 4 -volume $V \approx\left(\tau^{2} / 2\right) \Delta \eta V_{\perp}(b)$. In the latter, $\tau \approx 1 / 2 \mathrm{fm} / c$ is the prompt proper time over which the induced magnetic field is active, $\Delta \eta$ is the interval in pseudo-rapidity and $V_{\perp}(b)$ the transverse collision area for fixed impact parameter $b$. Through simple geometry

$$
V_{\perp}(b)=2 R^{2}\left(\arccos \left(\frac{b}{2 R}\right)-\frac{b}{2 R} \sqrt{1-\left(\frac{b}{2 R}\right)^{2}}\right)
$$

where $R$ is the radius of two identically colliding nuclei. $\mathbf{Q}^{2}$ involves a pair $\mathbf{P}, \mathbf{P}^{\prime}$ of instanton-antiinstanton. Specifically,

$$
\left\langle\mathbf{Q}^{2}\right\rangle_{V}=\left\langle\left(\mathbf{P}_{+}-\mathbf{P}_{-}\right)\left(\mathbf{P}_{+}^{\prime}-\mathbf{P}_{-}^{\prime}\right)\right\rangle_{V}
$$

If we denote by $N_{ \pm}$the number of instantons and antiinstantons in $V$, with $N=N_{+}+N_{-}$their total number, then in the instanton vacuum the pair correlation follows from

$$
\left\langle\mathbf{Q}^{2}\right\rangle_{V} \equiv\left\langle\left(\frac{N_{+}-N_{-}}{N_{+}+N_{-}}\right)^{2}\right\rangle_{V} \approx \frac{\left\langle\left(N_{+}-N_{-}\right)^{2}\right\rangle_{V}}{\left\langle\left(N_{+}+N_{-}\right)^{2}\right\rangle_{V}}
$$

Assuming $N_{ \pm}$to be large in $V$ it follows that 63

$$
\left\langle\mathbf{Q}^{2}\right\rangle_{V} \approx \frac{\langle N\rangle_{V}}{\langle N\rangle_{V}\left(\langle N\rangle_{V}+4 / \mathbf{b}\right)}
$$

The deviation from the Poissonian distribution in the variance of the number average reflects on the QCD trace anomaly in the instanton vacuum or $\left\langle N^{2}\right\rangle_{V}-\langle N\rangle_{V}^{2}=4 / \mathbf{b}\langle N\rangle_{V}$ and vanishes in the large $N_{c}$ limit 63 . Here $\mathbf{b}=11 N_{c} / 3$ is the coefficient of the 1-loop beta function $\beta\left(\rho_{c}\right) \approx \mathbf{b} / \ln \left(\Lambda \rho_{c}\right)$ (quenched). Thus

$$
\left\langle\mathbf{Q}^{2}\right\rangle_{V} \approx \frac{1}{n_{I}\left(\tau^{2} \Delta \eta V_{\perp}(b) / 2\right)+4 / \mathbf{b}}
$$

where we have used that the mean $\langle N\rangle_{V}=n_{I} V$ in the volume $V$. The topological fluctuations are suppressed by the collision 4 -volume. Note that we have ignored the role of the temperature on the topological fluctuations in peripheral 
collisions. Temperature will cause these topological fluctuations to deplete and vanish at the chiral transition point following the instanton-anti-instanton pairing [64. So our results will be considered as upper-bounds.

For simplicity, we will set $\langle x\rangle \approx 0.01$ for each parton and $\langle z\rangle \approx 0.5$. The measured multiplicity spectra in 65 at different centralities suggest $m_{\pi}\left\langle\left\langle K_{\perp}\right\rangle\left\langle 3 m_{\pi}\right.\right.$. We will set $\left\langle K_{\perp}\right\rangle=2 m_{\pi}$ in our analysis. We will assume a moderate polarization or $c \%=15 \%$ in the collision volume for a general discussion. We fix $\tau=1 / 2 \mathrm{fm}$ to be the maximum duration of the magnetic field polarization, and set the pseudo-rapidity interval approximately $(-1,1)$ for both STAR [59] and ALICE [62]. The radius of the colliding nuclei will be set to $R=1 \mathrm{fm} \times \sqrt[3]{A}$ where $A$ is the atomic number. The centrality is approximated as $n \%=b^{2} /(2 R)^{2}$ 66. Our results are displayed in Fig. 13 (left) for $A u A u$ and Fig. 13 (middle) for $C u C u$ collisions at $\sqrt{s}=200 \mathrm{GeV}$ (STAR), and in Fig. 13 (right) for $P b P b$ collisions at $\sqrt{s}=2.76 \mathrm{TeV}$ (ALICE). We recall that 67

$$
\left\langle\cos \left(\phi_{\alpha}+\phi_{\beta}-2 \Psi_{\mathrm{RP}}\right)\right\rangle \equiv-\left\langle a_{\alpha} a_{\beta}\right\rangle
$$

For the like-charges the results compare favorably with the data. For the unlike charges they overshoot the data especially for the heavier ion. Our results show a difference between $\pi^{+} \pi^{+}$and $\pi^{-} \pi^{-}$as we only retained the protons in our analysis. The inclusion of the neutrons would result into the same charge correlations for $\pi^{+} \pi^{+}$and $\pi^{-} \pi^{-}$by isospin symmetry.
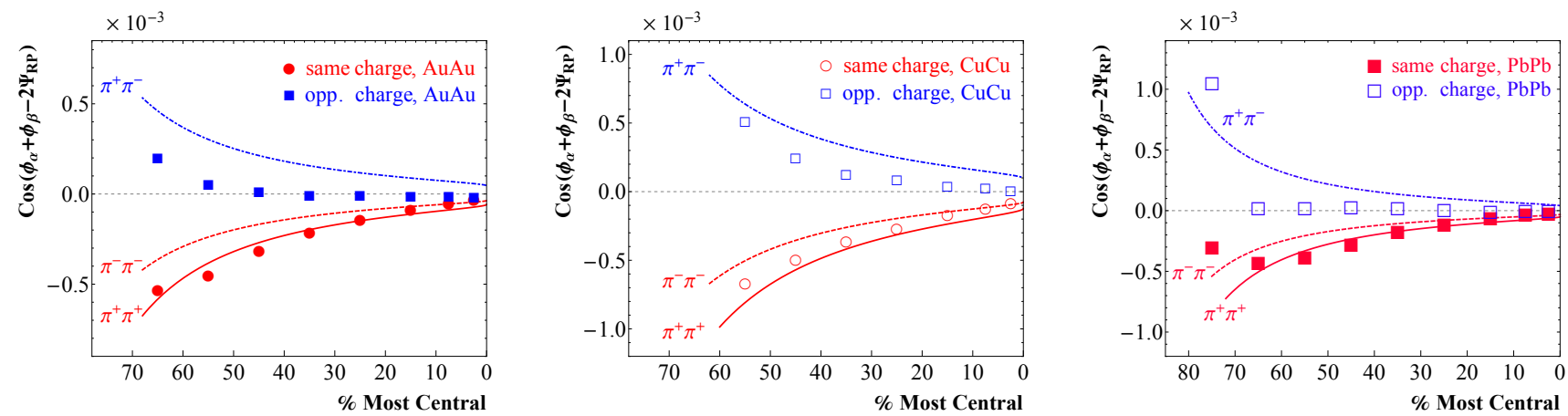

FIG. 13: Pion azimuthal charge correlations versus the data [59] from STAR at $\sqrt{s}=200 \mathrm{GeV}$ (left and middle) and the data from ALICE 62 at $\sqrt{s}=2.76 \mathrm{TeV}$ (right).

\section{CONCLUSIONS AND PROSPECTS}

Instantons and anti-instantons provide the key building blocks of the instanton liquid model. The latter offers a detailed framework for understanding aspects of the spontaneous breaking of chiral symmetry and the resolution of the $\mathrm{U}(1)$ problem. Key to this is the appearance of light quark zero modes of fixed chirality and their de-localization through the formation of an interacting liquid. Some aspects of this model are supported by lattice simulations upon cooling 3, 68, 69.

In light of the many phenomenological successes of the instanton liquid model, it is natural to ask about the role of instantons in scattering processes, in particular on spin physics. An essential aspect of the light quark zero modes is the emergence of large constituent masses and (chromo) magnetic moments. Also instantons and anti-instantons correlate strongly the spin with color leading to sizable contributions in spin polarized processes involving light quarks.

This review gives a brief summary of recent advances in the emerging field of spin physics where the induced effects by instantons and anti-instantons in a semi-classical analysis, are sizable in comparison to those usually parametrized using perturbation theory. We stress that the effects we have reported both in polarized electron-proton or protonproton semi-inclusive scattering, rely solely on the instanton liquid parameters in the vacuum without additional changes. The effects are large and comparable in size with those reported experimentally.

This review also shows that the large spin effects induced by instantons and anti-instantons in polarized experiments may also be present in peripheral $A A$ collisions where a prompt and large magnetic field can induce a prompt and large polarization although on a short time scale. A simple analysis of the correlated fluctuations between target and projectile protons shows that the effects is of the same magnitude and sign are those reported in the peripheral charged pion azimuthal correlations at collider energies. Again it is important to stress that only the fluctuations expected from instanton vacuum configurations were used. 


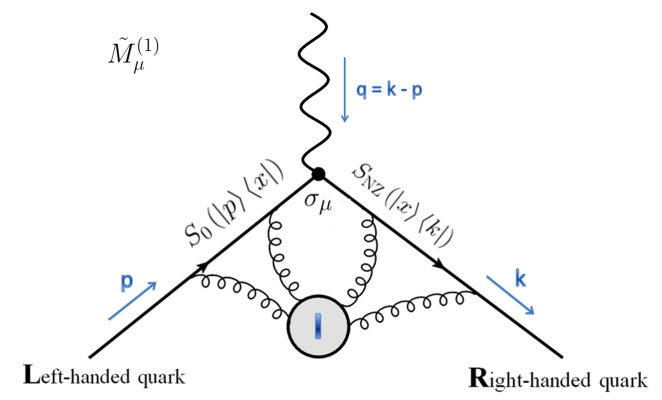

FIG. 14: The incoming left-handed quark with momentum $p$ meets one instanton and flips its chirality. The outgoing righthanded quark carries momentum $k$. The momentum of the photon is $q=p-k$. $S_{0}$ and $S_{n z}$ stand for the zero-mode quark propagator and the non-zero mode quark propagator in the single instanton background respectively.

This review is by no means exhaustive as many new effects can be explored using this framework. One important shortcoming of the instanton liquid model is the lack of confinement as described by an ordering of the eigenvalues of the Polyakov line at low temperature. Some important amendments to the instanton liquid model have been proposed, suggesting that instantons and anti-instantons split into dyons in the confined phase [70. It was recently shown that the key chiral effects and U(1) effects in the standard instanton liquid model are about similar to those emerging from the new instanton-dyon liquid model 71 77. It would be important to revisit the spin effects in this context.

\section{ACKNOWLEDGEMENTS}

This work was supported in parts by the US-DOE grant DE-FG-88ER40388.

\section{APPENDIX: EFFECTIVE VERTEX IN INSTANTON BACKGROUND}

\section{A. Photon vertex}

In this Appendix, we review the derivation of Eq. (23) in [28, corresponding to the nonperturbative insertion $\tilde{M}_{\mu}^{(1)}$ for photon exchange in the single instanton background. Similar calculations can also be found in [27, 39. According to [27, 37, 39, 40], the zero mode quark propagator in the single instanton background after Fourier transformation with respect to the incoming momentum $p$ is

$$
S_{0}(x, p)_{\dot{\beta}}^{j}{ }_{i \delta}^{j}=\frac{2 \rho^{2}}{\lambda} \frac{x^{l}\left(\bar{\sigma}_{l}\right)_{\dot{\beta} \gamma} \varepsilon^{\gamma j} \varepsilon_{i \delta}}{\left(x^{2}+\rho^{2}\right)^{\frac{3}{2}}|x|}
$$

Note the chirality of the zero mode flips as $|L\rangle\langle R|$ as depicted in Fig. 14. The incoming quark is left-handed and has momentum $p$ (on-shell). $\rho$ is the size of instanton and $\lambda$ is the mean virtuality. $\beta$ and $\delta$ are spatial indices, while $j$ and $i$ are color indices. In Euclidean space, $\sigma_{\mu}=(\vec{\sigma}, i I), \bar{\sigma}_{\mu}=(\vec{\sigma}, i I)$ and $\epsilon^{01}=-\epsilon^{10}=-\epsilon_{01}=\epsilon_{10}$ 32. The right-handed non-zero mode quark propagator in the single instanton after Fourier transformation with respect to the outgoing momentum $k$ is 27, 37, 39.

$$
S_{n z}(k, x)^{\beta i}{ }_{j \alpha}=-\delta_{\alpha}^{\beta}\left(\delta_{j}^{i}+\frac{\rho^{2}}{x^{2}} \frac{\left(\sigma_{\rho} \bar{\sigma}_{r}\right)^{i}{ }_{j} k^{\rho} x^{r}}{2 k \cdot x}\left(1-e^{-i k \cdot x}\right)\right) \frac{|x|}{\sqrt{x^{2}+\rho^{2}}} e^{i k \cdot x}
$$

Consider the process depicted in Fig. 14: the incoming left-handed quark meets one instanton and flips its chirality (zero-mode), then exchanges one photon, and finally becomes an outgoing right-handed quark. As a result, the nonperturbative insertion $M_{\mu}^{(1)}$ reads

$$
\left(\tilde{M}_{\mu}^{(1)}\right)_{i^{\prime} \delta}^{\beta i}=\int d^{4} x \quad e^{-i q \cdot x} S_{n z}(k, x)^{\beta i}{ }_{j \alpha} \sigma_{\mu}^{\alpha \dot{\beta}} S_{0}(x, p)_{\dot{\beta}}^{j}{ }_{i^{\prime} \delta}
$$


All the other parts of the diagram are trivial in color, therefore we take the trace of color indices $i$ and $i^{\prime}$. To further simplify the result, we need the following formula 32

$$
\begin{gathered}
\delta_{\alpha}^{\beta} \delta_{j}^{i}\left(\sigma_{\mu}\right)^{\alpha \dot{\beta}}\left(\bar{\sigma}_{l}\right)_{\dot{\beta} \gamma} \varepsilon^{\gamma j} \varepsilon_{i \delta}=\left(\sigma_{\mu} \bar{\sigma}_{l}\right)_{\delta}^{\beta} \\
\delta_{\alpha}^{\beta}\left(\sigma_{\rho} \bar{\sigma}_{r}\right)^{i}{ }_{j}\left(\sigma_{\mu}\right)^{\alpha \dot{\beta}}\left(\bar{\sigma}_{l}\right)_{\dot{\beta} \gamma} \varepsilon^{\gamma j} \varepsilon_{i \delta}=\left(\sigma_{\mu} \bar{\sigma}_{l} \sigma_{r} \bar{\sigma}_{\rho}\right)^{\beta}{ }_{\delta}
\end{gathered}
$$

Combining all the equations above, we obtain

$$
\tilde{M}_{\mu}^{(1)}=-\int d^{4} x \quad\left(\frac{2 \rho^{2}}{\lambda} \sigma_{\mu} \bar{\sigma}_{l} e^{i p \cdot x} \frac{x^{l}}{\left(x^{2}+\rho^{2}\right)^{2}}+\sigma_{\mu} \bar{\sigma}_{\rho} k^{\rho} \frac{\rho^{4}}{\lambda}\left(e^{i p \cdot x}-e^{-i q \cdot x}\right) \frac{1}{\left(x^{2}+\rho^{2}\right)^{2}(k \cdot x)}\right)
$$

The $d^{4} x$ integration in Eq. 82 can be done with the help of the following formula $\left(p^{2} \longrightarrow 0\right)$

$$
\begin{gathered}
\int d^{4} x e^{i p \cdot x} \frac{x^{l}}{\left(x^{2}+\rho^{2}\right)^{2}}=i 2 \pi^{2} \frac{p^{l}}{p^{2}} \\
\int d^{4} x \frac{e^{i p \cdot x}}{\left(x^{2}+\rho^{2}\right)^{2}(k \cdot x)}=-i \frac{\pi^{2}}{p \cdot k} \frac{\rho|p|}{\rho^{2}} K_{1}(\rho|p|)=i \frac{2 \pi^{2}}{q^{2}} \frac{\rho|p|}{\rho^{2}} K_{1}(\rho|p|)
\end{gathered}
$$

where we used $-2 p \cdot k=(k-p)^{2}-k^{2}-p^{2} \approx q^{2}$. In our paper [28, we explicitly showed that all terms proportional to $\bar{\sigma}_{l} p^{l}$ vanish. Thus

$$
\tilde{M}_{\mu}^{(1)}=-i \frac{4 \pi^{2} \rho^{2}}{\lambda} \sigma_{\mu} \bar{\sigma}_{l} \frac{k^{l}}{q^{2}}[f(\rho|p|)-f(\rho|q|)]
$$

where $f(a)=a K_{1}(a)$ As the incoming quark with momentum $p$ is on-shell and the mass of the quark is small $\left(p^{2} \longrightarrow 0\right)$, we have

$$
f(\rho|p|)=\rho|p| K_{1}(\rho|p|) \longrightarrow \rho|p| \frac{1}{\rho|p|}=1
$$

Since $q^{2}<0$ in SIDIS, we define $Q^{2}=-q^{2}>0$. Eq. 85 simplifies to

$$
\tilde{M}_{\mu}^{(1)}=i \frac{4 \pi^{2} \rho^{2}}{\lambda} \sigma_{\mu} \bar{\sigma}_{l} \frac{k^{l}}{Q^{2}}[1-f(\rho Q)]
$$

Here we note that Eq. (87) is derived from Eq. (79) which pictorially reads

- Left-handed quark $(\vec{p}) \stackrel{\text { Instanton }}{\longrightarrow}$ Right-handed quark (zero mode) $\stackrel{\text { Photon }(\vec{q})}{\longrightarrow}$ Right-handed quark $(\vec{k})$ where $\vec{q}=\vec{k}-\vec{p}$. On the other hand, if we consider

- Right-handed quark $(\vec{k}) \stackrel{\text { Photon }(-\vec{q})}{\longrightarrow}$ Right-handed quark (zero mode) $\stackrel{\text { Anti-instanton }}{\longrightarrow}$ Left-handed quark $(\vec{p})$ instead of Eq. (87), we would obtain

$$
\tilde{M}_{\mu}^{(1)}=-i \frac{4 \pi^{2} \rho^{2}}{\lambda^{\dagger}} \sigma_{\mu} \bar{\sigma}_{l} \frac{k^{l}}{Q^{2}}[1-f(\rho Q)]
$$

where we have taken the conjugate of Eq. (87) and replaced $p \leftrightarrow k$. In [28] we used the replacement $k \leftrightarrow-p$. We have checked that our final results are left unchanged by this correction. Thus

$$
M_{\mu}^{(1)}=4 \pi^{2} \rho^{2}\left(i \frac{\mathbf{P}_{+}}{\lambda} \sigma_{\mu} \bar{\sigma}_{l} k^{l}-i \frac{\mathbf{P}_{-}}{\lambda^{\dagger}} \sigma_{l} \bar{\sigma}_{\mu} p^{l}\right) \frac{[1-f(\rho Q)]}{Q^{2}}
$$

where $\mathbf{P}_{ \pm}=1 / 0$ denote one or no instanton/anti-instanton. Similarly, for the processes depicted pictorially as 
- Right-handed quark $(\vec{p}) \stackrel{\text { Anti-Instanton }}{\longrightarrow}$ Left-handed quark (zero mode) $\stackrel{\text { Photon }(\vec{q})}{\longrightarrow}$ Left-handed quark $(\vec{k})$

- Left-handed quark $(\vec{k}) \stackrel{\text { Photon }(-\vec{q})}{\longrightarrow}$ Left-handed quark (zero mode) $\stackrel{\text { Instanton }}{\longrightarrow}$ Right-handed quark $(\vec{p})$

Thus the result combining both the instanton and anti-instanton contributions

$$
M_{\mu}^{(1)}=4 \pi^{2} \rho^{2}\left(i \frac{\mathbf{P}_{+}}{\lambda} \gamma_{\mu} \not k-i \frac{\mathbf{P}_{-}}{\lambda^{\dagger}} \not p \gamma_{\mu}\right) \frac{[1-f(\rho Q)]}{Q^{2}}
$$

Now, we need to average Eq. (90) using the instanton liquid model. The standard averaging in the vacuum is

$$
\left\langle\frac{1}{\lambda}\right\rangle=\frac{1}{2 N} \int \frac{d \lambda}{\lambda} n(\lambda)=-i \pi \frac{n(0)}{N}
$$

where by Banks-Casher relation is used $\pi n(0) / N=-\left\langle q^{\dagger} q\right\rangle$. However, we note that $\mathbf{P}_{ \pm}$in Eq. (90) means that we fix an instanton or anti-instanton pertaining to the polarized hadron prior to the averaging. This means that the pertinent eigenvalue distribution instead is

$$
n( \pm, \lambda)=n(\lambda)-\delta\left(\lambda \mp \lambda_{*} / N\right)
$$

with $\lambda_{*}$ a typical eigenvalue in the zero-mode-zone. Technically $n( \pm, \lambda)$ amounts to fixing an instanton or antiinstanton, and averaging over the remainder of the instanton-antiinstanton liquid by removing 1-row and 1-column in the $N \times N$ overlap matrix of zero-modes $\mathbf{T}_{\mathbf{I J}}$ for the fixed instanton or anti-instanton while averaging with detT in the instanton liquid model. Explictly, this amounts to

$$
\frac{\mathbf{P}_{ \pm}}{\lambda} \stackrel{\text { fix an instanton/anti-instanton }}{\longrightarrow}\left\langle\frac{\mathbf{P}_{ \pm}}{\lambda}\right\rangle=\frac{1}{N} \int \frac{d \lambda}{\lambda} n( \pm, \lambda)=\mp \frac{1}{\lambda_{*}}-i \pi \frac{n(0)}{N}
$$

Thus

$$
\left\langle M_{\mu}^{(1)}\right\rangle=4 \pi^{2} \rho^{2} \frac{\pi n(0)}{N}\left(\gamma_{\mu} \not k+\not p \gamma_{\mu}\right) \frac{[1-f(\rho Q)]}{Q^{2}}-i \frac{4 \pi^{2} \rho^{2}}{\lambda_{*}}\left(\gamma_{\mu} \not k+\not p \gamma_{\mu}\right) \frac{[1-f(\rho Q)]}{Q^{2}}
$$

The real part can be re-written as

$$
\begin{aligned}
4 \pi^{2} \rho^{2} \frac{\pi n(0)}{N}\left(\gamma_{\mu} \not k+\not p \gamma_{\mu}\right) \frac{[1-f(\rho Q)]}{Q^{2}} & =4 \pi^{2} \rho^{2} \frac{\pi n(0)}{N} \frac{[1-f(\rho Q)]}{Q^{2}} q^{\nu} \sigma_{\mu \nu}+4 \pi^{2} \rho^{2} \frac{\pi n(0)}{N} \frac{[1-f(\rho Q)]}{Q^{2}}\left(\not k \gamma_{\mu}-\gamma_{\mu} \not p\right) \\
& \longrightarrow 4 \pi^{2} \rho^{2} \frac{\pi n(0)}{N} \frac{[1-f(\rho Q)]}{Q^{2}} q^{\nu} \sigma_{\mu \nu}
\end{aligned}
$$

where $\sigma_{\mu \nu}=\left[\gamma_{\mu}, \gamma_{\nu}\right]$. The parts proportional to $\not k \gamma_{\mu}$ and $\gamma_{\mu} \not p$ vanish as discussed in [78. The imaginary part is

$$
-i \frac{4 \pi^{2} \rho^{2}}{\lambda_{*}}\left(\gamma_{\mu} \not k+\not p \gamma_{\mu}\right) \frac{[1-f(\rho Q)]}{Q^{2}}
$$

and contributes to SSA in SIDIS as noted in [27, 28].

\section{B. Gluon vertex}

The QCD vacuum is a random ensemble of instantons and anti-instantons interacting via the exchange of perturbative gluons and quasi-zero modes of light quarks and anti-quarks. In the dilute instanton approximation, a typical effective vertex with quarks and gluons attached to an instanton is shown in Fig. 15. The corresponding effective vertex is given by 79 , 


$$
\mathcal{L}=\int \prod_{q}\left[m_{q} \rho-2 \pi^{2} \rho^{3} \bar{q}_{R}\left(1+\frac{i}{4} \tau^{a} \bar{\eta}_{\mu \nu}^{a} \sigma_{\mu \nu}\right) q_{L}\right] \exp \left(-\frac{2 \pi^{2}}{g_{s}} \rho^{2} \bar{\eta}_{\gamma \delta}^{b} G_{\gamma \delta}^{b} F_{g}(\rho Q)\right) d_{0}(\rho) \frac{d \rho}{\rho^{5}} d \bar{\sigma}+(L \leftrightarrow R)
$$

where $d \bar{\sigma}$ is the integration over the instanton orientation in color space and $\sigma_{\mu \nu}=\left[\gamma_{\mu}, \gamma_{\nu}\right] / 2$. The incoming and outgoing quarks have small momenta $p(\rho p \ll 1)$ and $Q$ is the momentum transferred by the inserted gluon with a form-factor

$$
F_{g}(x) \equiv \frac{4}{x^{2}}-2 K_{2}(x) \stackrel{x \rightarrow 0}{\longrightarrow} 1
$$

By expanding Eq. (97) to leading order in the inserted gluon field of $G_{\gamma \delta}^{b}$ and integrating over the color indices, we obtain

$$
\frac{i}{g_{s}} F_{g}(\rho Q) \int \pi^{4} \rho^{4} \frac{\bar{q}_{R} t^{a} \sigma_{\mu \nu} q_{L}}{m_{q}^{*}} G_{\mu \nu}^{a} \times\left(\prod_{q}\left(\rho m_{q}^{*}\right) d_{0}(\rho) \frac{d \rho}{\rho^{5}}\right)=\frac{i}{g_{s}} F_{g}(\rho Q) \int d \rho \pi^{4} \rho^{4} n(\rho) \frac{\bar{q}_{R} t^{a} \sigma_{\mu \nu} q_{L}}{m_{q}^{*}} G_{\mu \nu}^{a}
$$

where $n(\rho)$ is the effective instanton density and $m_{q}^{*}$ is the effective quark mass. In the dilute instanton approximation 36 .

$$
n(\rho)=n_{I} \delta\left(\rho-\rho_{c}\right)
$$

where $\rho_{c}$ is the average size of the instanton. Hence the induced instanton effective quark-gluon vertex

$$
\frac{i}{g_{s}} F_{g}(\rho Q) \pi^{4}\left(n_{I} \rho_{c}^{4}\right) \frac{\bar{q}_{R} t^{a} \sigma_{\mu \nu} q_{L}}{m_{q}^{*}} G_{\mu \nu}^{a}
$$

as illustrated in Fig. 15. In momentum space, the effective vertex is $M_{\mu}^{a}$ and reads

$$
M_{\mu}^{a}=t^{a}\left[\gamma_{\mu}-\mathbf{P}_{+} \gamma_{+} \sigma_{\mu \nu} q^{\nu} \Psi-\mathbf{P}_{-} \gamma_{-} \sigma_{\mu \nu} q^{\nu} \Psi\right]
$$

with $\gamma_{ \pm}=\left(1 \pm \gamma_{5}\right) / 2$ and

$$
\Psi=\frac{F_{g}\left(\rho_{c} Q\right) \pi^{4}\left(n_{I} \rho_{c}^{4}\right)}{m_{q}^{*} g_{s}^{2}}
$$

The averaging of Eq. 102 in the instanton liquid gives

$$
\left\langle M_{\mu}^{a}\right\rangle=t^{a}\left[\gamma_{\mu}-\sigma_{\mu \nu} q^{\nu} \Psi\right]
$$

where we used

$$
\left\langle\mathbf{P}_{+}\right\rangle=\left\langle\mathbf{P}_{-}\right\rangle=1
$$

after the analytical continuation to Minkowski Space. Eq. 101) yields an anomalously large Quark Chromomagnetic Moment 81]

$$
\mu_{a}=-\frac{2 n_{I} \pi^{4} \rho_{c}^{4}}{g_{s}^{2}}
$$




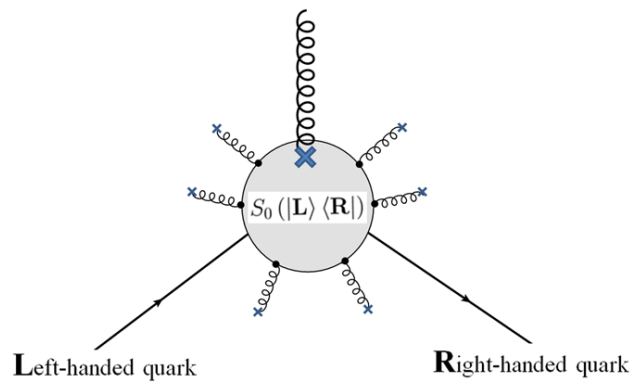

FIG. 15: Effective Quark-Gluon vertex in the instanton vacuum.

\section{Kinematics revisited}

The simplifying assumption $\left\langle x_{1}\right\rangle=\left\langle x_{2}\right\rangle$ may appear to be unrealistic especially for large $x_{F}$. Here, we improve on this simplification and show that this amounts to a better fit to the data. In the lab frame, we define

$$
\begin{aligned}
& p_{1}=x_{1} \frac{\sqrt{s}}{2}(1,0,0,1) \\
& p_{2}=x_{2} \frac{\sqrt{s}}{2}(1,0,0,-1)
\end{aligned}
$$

set

$$
\tilde{s}=\frac{s}{4}\left[\left(x_{1}+x_{2}\right)^{2}-\left(x_{1}-x_{2}\right)^{2}\right]=x_{1} x_{2} s
$$

and identify the two Lorentz factors

$$
\gamma=\frac{x_{1}+x_{2}}{2 \sqrt{x_{1} x_{2}}}, \quad \text { and } \quad \beta=\frac{x_{1}-x_{2}}{x_{1}+x_{2}}
$$

The longitudinal momentum of the outgoing particle $k_{1}$ is

$$
k_{L}=\frac{\sqrt{s}}{2} x_{F}=\gamma\left(k_{L}^{*}+\beta E^{*}\right)
$$

where $k_{L}^{*}$ is the longitudinal momentum of the particle $k_{1}$ in the CM frame and $E^{*}$ is the energy of particle $k_{1}$ in the CM frame. We have

$$
k_{L}^{*}=\frac{\sqrt{\tilde{s}}}{2} \cos \theta, \quad \text { and } \quad E^{*}=\frac{\sqrt{\tilde{s}}}{2}
$$

with

$$
\cos \theta=\frac{2 x_{F}-x_{1}+x_{2}}{x_{1}+x_{2}}
$$

Also we have

$$
k^{\perp}=\frac{\sqrt{\tilde{s}}}{2} \sin \theta, \quad \text { and } \quad Q=\sqrt{\tilde{s}} \sin \left(\frac{\theta}{2}\right)
$$

With this in mind and for $x_{F}=0.6$, we show in Fig. 16 the dependence of the SSA $A_{T}$ on $x_{1} \in(0.6,0.9)$ and $x_{2} \in(0.1,0.3)$ for charged and uncharged pions. 

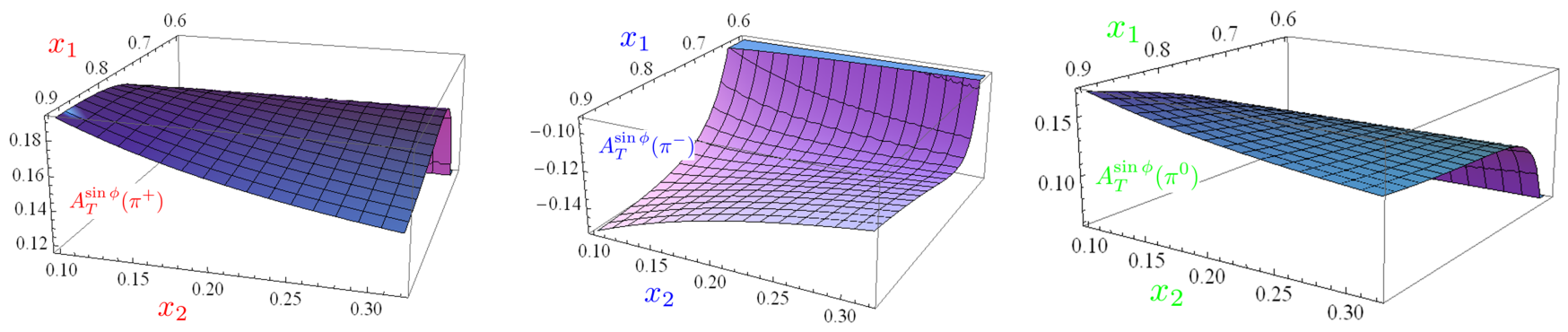

FIG. 16: $\quad \operatorname{SSAs} A_{T}^{\sin \phi}\left(\pi^{+}\right), A_{T}^{\sin \phi}\left(\pi^{-}\right)$, and $A_{T}^{\sin \phi}\left(\pi^{0}\right)$ for $x_{F}=0.6$.

Take $A_{T}^{\sin \phi}\left(\pi^{+}\right)$for example, the SSA $A_{T}^{\sin \phi}\left(\pi^{+}\right)$increases when $x_{1}$ increases and $x_{2}$ decreases. The simplifying assumption $\left\langle x_{1}\right\rangle=\left\langle x_{2}\right\rangle$, overestimates $x_{1}$ and underestimates $x_{2}$ for small $x_{F}$. Also, we underestimate $x_{1}$ and overstimate $x_{2}$ for large $x_{F}$. Instead of using $\left\langle x_{1}\right\rangle=\left\langle x_{2}\right\rangle$, a more careful calcution shows that $A_{T}^{\sin \phi}\left(\pi^{+}\right)$is smaller for small $x_{F}$ and larger for large $x_{F}$, and therefore fits the data better. Again, in this paper our aim was to show that instanton do contribute sizably to SSA with the right magnitude and trend. They have to be included in any perturbative analysis of these effects.

[1] D. B. Leinweber, J. I. Skullerud, A. G. Williams, and C. Parrinello (UKQCD), Phys. Rev. D60, 094507 (1999), [Erratum: Phys. Rev.D61,079901(2000)], hep-lat/9811027.

[2] D. B. Leinweber, A. W. Thomas, and R. D. Young, Phys. Rev. Lett. 92, 242002 (2004), hep-lat/0302020.

[3] M. C. Chu, J. M. Grandy, S. Huang, and J. W. Negele, Phys. Rev. D49, 6039 (1994), hep-lat/9312071.

[4] T. Schfer and E. V. Shuryak, Rev.Mod.Phys. 70, 323 (1998), hep-ph/9610451.

[5] M. Nowak, M. Rho, and I. Zahed, Chiral Nuclear Dynamics, v. 1 (World Scientific Publishing Company, Incorporated, 1996), ISBN 9789810210007, URL http://books.google.com/books?id=zhd2QgAACAAJ

[6] E. V. Shuryak and I. Zahed, Phys. Rev. D62, 085014 (2000), hep-ph/0005152.

[7] M. A. Nowak, E. V. Shuryak, and I. Zahed, Phys. Rev. D64, 034008 (2001), hep-ph/0012232.

[8] E. V. Shuryak and I. Zahed, Phys. Rev. D69, 014011 (2004), hep-ph/0307103.

[9] D. Kharzeev and E. Levin, Nucl. Phys. B578, 351 (2000), hep-ph/9912216.

[10] A. Dorokhov and I. Cherednikov, Nucl.Phys.Proc.Suppl. 146, 140 (2005), hep-ph/0412082.

[11] A. Ringwald and F. Schrempp, Phys. Lett. B503, 331 (2001), hep-ph/0012241.

[12] F. Schrempp and A. Utermann, in 5th Internationa Conference on Strong and Electroweak Matter (SEWM 2002) Heidelberg, Germany, October 2-5, 2002 (2003), hep-ph/0301177.

[13] M. Giordano and E. Meggiolaro, Phys.Rev. D81, 074022 (2010), 0910.4505.

[14] M. Giordano and E. Meggiolaro, PoS LATTICE2011, 155 (2011), 1110.5188.

[15] A. Airapetian et al. (HERMES), Phys. Rev. Lett. 94, 012002 (2005), hep-ex/0408013.

[16] A. Airapetian et al. (HERMES), Phys. Rev. Lett. 103, 152002 (2009), 0906.3918.

[17] H. Avakian et al. (CLAS), Phys. Rev. Lett. 105, 262002 (2010), 1003.4549.

[18] B. Abelev et al. (STAR Collaboration), Phys.Rev.Lett. 101, 222001 (2008), 0801.2990.

[19] K. Eyser (PHENIX Collaboration), AIP Conf.Proc. 842, 404 (2006).

[20] D. L. Adams et al. (FNAL-E704), Phys. Lett. B264, 462 (1991).

[21] D. W. Sivers, Phys. Rev. D41, 83 (1990).

[22] D. W. Sivers, Phys. Rev. D43, 261 (1991).

[23] J. C. Collins, Nucl. Phys. B396, 161 (1993), hep-ph/9208213.

[24] J. C. Collins, S. F. Heppelmann, and G. A. Ladinsky, Nucl. Phys. B420, 565 (1994), hep-ph/9305309.

[25] N. Kochelev, JETP Lett. 72, 481 (2000), hep-ph/9905497.

[26] A. E. Dorokhov, N. I. Kochelev, and W. D. Nowak, Phys. Part. Nucl. Lett. 6, 440 (2009), 0902.3165.

[27] D. Ostrovsky and E. Shuryak, Phys.Rev. D71, 014037 (2005), hep-ph/0409253.

[28] Y. Qian and I. Zahed, Phys.Rev. D86, 014033 (2012), 1112.4552.

[29] N. Kochelev and N. Korchagin, Phys.Lett. B729, 117 (2014), 1308.4857.

[30] S. J. Brodsky, D. S. Hwang, Y. V. Kovchegov, I. Schmidt, and M. D. Sievert, Phys. Rev. D88, 014032 (2013), 1304.5237.

[31] A. Metz, D. Pitonyak, A. Schfer, M. Schlegel, W. Vogelsang, and J. Zhou, Few Body Syst. 56, 331 (2015).

[32] S. Vandoren and P. van Nieuwenhuizen (2008), 0802.1862.

[33] R. Jackiw and C. Rebbi, Phys. Rev. Lett. 37, 172 (1976).

[34] C. G. Callan, Jr., R. F. Dashen, and D. J. Gross, Phys. Lett. B63, 334 (1976).

[35] A. M. Polyakov, Nucl. Phys. B120, 429 (1977).

[36] E. V. Shuryak, Nucl. Phys. B198, 83 (1982). 
[37] L. S. Brown, R. D. Carlitz, D. B. Creamer, and C.-k. Lee, Phys.Rev. D17, 1583 (1978).

[38] N. Andrei and D. J. Gross, Phys. Rev. D18, 468 (1978).

[39] S. Moch, A. Ringwald, and F. Schrempp, Nucl.Phys. B507, 134 (1997), hep-ph/9609445.

[40] P. Faccioli and E. V. Shuryak, Phys.Rev. D64, 114020 (2001), hep-ph/0106019.

[41] Y. Qian and I. Zahed, Phys. Rev. D90, 114012 (2014), 1404.6270.

[42] Y. Qian and I. Zahed, Nucl. Phys. A940, 227 (2015), 1205.2366.

[43] E. V. Shuryak and I. Zahed, Phys. Rev. D70, 054507 (2004), hep-ph/0403127.

[44] A. Airapetian et al. (HERMES), Phys. Lett. B442, 484 (1998), hep-ex/9807015.

[45] B. Potter (1997).

[46] M. Hirai, S. Kumano, and N. Saito, Phys.Rev. D74, 014015 (2006), hep-ph/0603213.

[47] D. Adams et al. (Fermilab E704 Collaboration), Nucl.Phys. B510, 3 (1998).

[48] J. Skeens (E704 Collaboration), AIP Conf.Proc. 243, 1008 (1992).

[49] M. Hutter (1993), hep-ph/9501335.

[50] I. Arsene et al. (BRAHMS), Phys. Rev. Lett. 101, 042001 (2008), 0801.1078.

[51] A. Adare et al. (PHENIX), Phys. Rev. D90, 012006 (2014), 1312.1995.

[52] J. Beringer et al. (Particle Data Group), Phys. Rev. D86, 010001 (2012).

[53] V. Skokov, A. Y. Illarionov, and V. Toneev, Int.J.Mod.Phys. A24, 5925 (2009), 0907.1396.

[54] D. Kharzeev, R. Pisarski, and M. H. Tytgat, Phys.Rev.Lett. 81, 512 (1998), hep-ph/9804221.

[55] D. Kharzeev, Phys.Lett. B633, 260 (2006), hep-ph/0406125.

[56] D. E. Kharzeev, L. D. McLerran, and H. J. Warringa, Nucl.Phys. A803, 227 (2008), 0711.0950.

[57] K. Fukushima, D. E. Kharzeev, and H. J. Warringa, Phys.Rev. D78, 074033 (2008), 0808.3382.

[58] G. Basar, G. V. Dunne, and D. E. Kharzeev, Phys.Rev. D85, 045026 (2012), 1112.0532.

[59] B. Abelev et al. (STAR Collaboration), Phys.Rev.Lett. 103, 251601 (2009), 0909.1739.

[60] B. Abelev et al. (STAR Collaboration), Phys.Rev. C81, 054908 (2010), 0909.1717.

[61] P. Christakoglou, J.Phys. G38, 124165 (2011), 1106.2826.

[62] I. Selyuzhenkov (ALICE Collaboration), PoS WPCF2011, 044 (2011), 1203.5230.

[63] D. Diakonov and V. Y. Petrov, Nucl.Phys. B245, 259 (1984).

[64] R. A. Janik, M. A. Nowak, G. Papp, and I. Zahed, AIP Conf.Proc. 494, 408 (1999), hep-lat/9911024.

[65] P. K. Khandai, P. Shukla, and V. Singh, Phys. Rev. C84, 054904 (2011), 1110.3929.

[66] C. Aguiar, T. Kodama, R. Andrade, F. Grassi, Y. Hama, et al., Braz.J.Phys. 34, 319 (2004).

[67] S. A. Voloshin, Phys.Rev. C70, 057901 (2004), hep-ph/0406311.

[68] M. C. Chu, J. M. Grandy, S. Huang, and J. W. Negele, Phys. Rev. Lett. 70, 255 (1993), hep-lat/9211019.

[69] M. C. Chu, J. M. Grandy, S. Huang, and J. W. Negele, Phys. Rev. D48, 3340 (1993), hep-lat/9306002.

[70] D. Diakonov and V. Petrov, Phys. Rev. D76, 056001 (2007), 0704.3181.

[71] E. Shuryak and T. Sulejmanpasic, Phys. Rev. D86, 036001 (2012), 1201.5624.

[72] E. Shuryak, Nucl. Phys. A928, 138 (2014), 1401.2032.

[73] R. Larsen and E. Shuryak (2014), 1408.6563.

[74] R. Larsen and E. Shuryak, Phys. Rev. D92, 094022 (2015), 1504.03341.

[75] R. Larsen and E. Shuryak (2015), 1511.02237.

[76] Y. Liu, E. Shuryak, and I. Zahed, Phys. Rev. D92, 085006 (2015), 1503.03058.

[77] Y. Liu, E. Shuryak, and I. Zahed, Phys. Rev. D92, 085007 (2015), 1503.09148.

[78] N. Kochelev, Phys.Lett. B565, 131 (2003), hep-ph/0304171.

[79] G. 't Hooft, Phys.Rev. D14, 3432 (1976).

[80] A. Vainshtein, V. I. Zakharov, V. Novikov, and M. A. Shifman, Sov.Phys.Usp. 25, 195 (1982).

[81] N. Kochelev, Phys.Lett. B426, 149 (1998), hep-ph/9610551. 